\title{
Regenerating hyperbolic cone structures from Nil
}

\author{
JOAN PORTI \\ Departament de Matemàtiques, Universitat Autònoma de Barcelona \\ 08193 Bellaterra, Spain \\ Email: porti@mat.uab.es
}

\begin{abstract}
Let $\mathcal{O}$ be a three-dimensional $N i l$-orbifold, with branching locus a knot $\Sigma$ transverse to the Seifert fibration. We prove that $\mathcal{O}$ is the limit of hyperbolic cone manifolds with cone angle in $(\pi-\varepsilon, \pi)$. We also study the space of Dehn filling parameters of $\mathcal{O}-\Sigma$. Surprisingly it is not diffeomorphic to the deformation space constructed from the variety of representations of $\mathcal{O}-\Sigma$. As a corollary of this, we find examples of spherical cone manifolds with singular set a knot that are not locally rigid. Those examples have large cone angles.
\end{abstract}

AMS Classification numbers Primary: 57M10

Secondary: $58 \mathrm{M} 15$

Keywords: Hyperbolic structure, cone 3-manifolds, local rigidity

Proposed: Jean-Pierre Otal

Seconded: David Gabai, Walter Neumann
Received: 16 July 2001

Revised: 9 December 2002

(c) Geometry $\mathcal{G}$ Topology $\mathcal{P}$ ublications 


\section{Introduction}

This paper is motivated by a phenomenon occurring in the proof of the orbifold theorem. This proof suggests that some orbifolds with geometry $N i l$ appear as limit of rescaled hyperbolic cone manifolds. In the current proofs of the orbifold theorem $[4,5,6,3,8]$, it is only shown that those families of cone manifolds collapse, and this is used to construct a Seifert fibration of the orbifold, without knowing which kind of geometric structure is involved.

Every closed three-dimensional Nil orbifold admits an orbifold Seifert fibration. We assume that the ramification locus is a circle transverse to its Seifert fibration. This implies that the ramification index is 2 . Hence we view the orbifold as a cone manifold with cone angle $\pi$.

Theorem A Let $\mathcal{O}$ be a closed three-dimensional Nil orbifold whose ramification locus $\Sigma$ is a circle transverse to its Seifert fibration. Then there exist a family of hyperbolic cone structures on the underlying space of $\mathcal{O}$ with singular set $\Sigma$ parametrized by the cone angle $\alpha \in(\pi-\varepsilon, \pi)$, for some $\varepsilon>0$.

In addition, when $\alpha \rightarrow \pi^{-}$these hyperbolic cone manifolds converge to a point. If they are re-scaled by $(\pi-\alpha)^{-1 / 3}$, then they converge to a Euclidean 2 -orbifold, which is the basis of the Seifert fibration of $\mathcal{O}$. Finally, if they are re-scaled by $(\pi-\alpha)^{-1 / 3}$ in the horizontal direction and $(\pi-\alpha)^{-2 / 3}$ in the vertical one, then they converge to $\mathcal{O}$.

If the ramification locus $\Sigma$ was a circle but not transverse to the Seifert fibration of $\mathcal{O}$, then $\Sigma$ would be a fibre. In this case the conclusion of Theorem A could not hold, because $\mathcal{O}-\Sigma$ must be hyperbolic, and therefore $\mathcal{O}-\Sigma$ can not be Seifert fibred.

The following corollary follows from Theorem A and Kojima's global rigidity theorem [14].

Corollary 1.1 Let $\mathcal{O}$ be an orbifold as in Theorem A. There exist a family of hyperbolic cone structures on the underlying space of $\mathcal{O}$ with singular set $\Sigma$ parametrized by the cone angle $\alpha \in(0, \pi)$.

The first part of Theorem A is a particular case of Theorem B below, which gives a larger space of deformations parametrized by Dehn-filling coefficients. A cone manifold structure on $|\mathcal{O}|$ with singular set $\Sigma$ induces a non-complete metric on $\mathcal{O}-\Sigma$, whose completion is precisely the cone manifold. This is a 
particular case of structures on the end of $\mathcal{O}-\Sigma$ called of Dehn type. Those structures are defined by Thurston in [20] and they are described by a pair $(p, q) \in \mathbb{R}^{2} \cup\{\infty\}$.

Theorem B Let $\mathcal{O}$ be a Nil 3-orbifold as in Theorem A. There exists a neighborhood $U$ of $(2,0)$ in $\mathbb{R}^{2}$ and two $\mathcal{C}^{1}$-functions $f:(-\varepsilon, \varepsilon) \rightarrow(-\infty, 2]$ concave and $g:(-\varepsilon, \varepsilon) \rightarrow[2,+\infty)$ convex, with $\left.\left.f\right|_{[0, \varepsilon)} \equiv g\right|_{[0, \varepsilon)} \equiv 2$ and

$$
\lim _{q \rightarrow 0^{-}} \frac{2-f(q)}{|q|^{3 / 2}}=\lim _{q \rightarrow 0^{-}} \frac{g(q)-2}{|q|^{3 / 2}}>0,
$$

such that the following hold. Every point in $\{(p, q) \in U \mid p \geq f(q)\}$ is the Dehn-filling coefficient of a geometric structure on $\mathcal{O}-\Sigma$ of the following kind:

- hyperbolic for $p>g(q)$;

- Euclidean for $p=g(q), q<0$;

- spherical for $p<g(q), q>0$.

In addition, every point in the line $p=2$ corresponds to a transversely Riemannian foliation of codimension two (transversely hyperbolic for $q>0$, Euclidean for $q=0$ and spherical for $q>0)$.

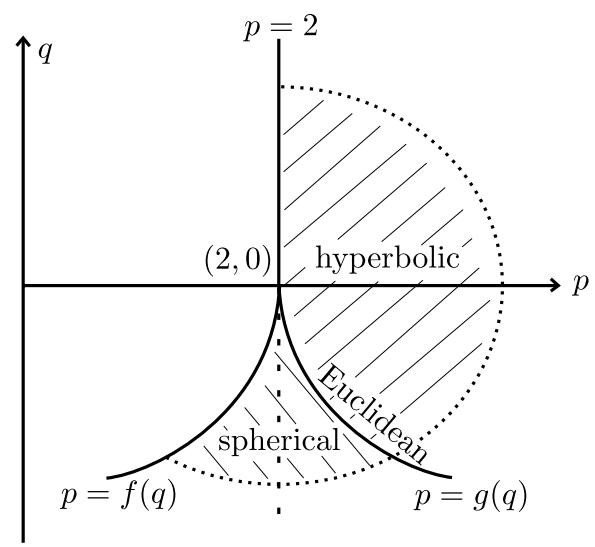

Figure 1: The open set of Theorem B

When $q=0$, Dehn filling coefficients $(p, 0)$ correspond to cone structures with cone angle $2 \pi / p$. Hence Theorem B implies the existence of hyperbolic cone manifolds with cone angles in $(\pi-\varepsilon, \pi)$ of Theorem A.

To prove Theorem B, we construct a deformation space homeomorphic to a half-disc. However, Dehn filling coefficients do not define a homeomorphism 
between the deformation space and the region of Theorem $\mathrm{B}$, because there is a Whitney pleat at the point $(p, q)=(2,0)$ corresponding to the Nil structure (see Figure 2).
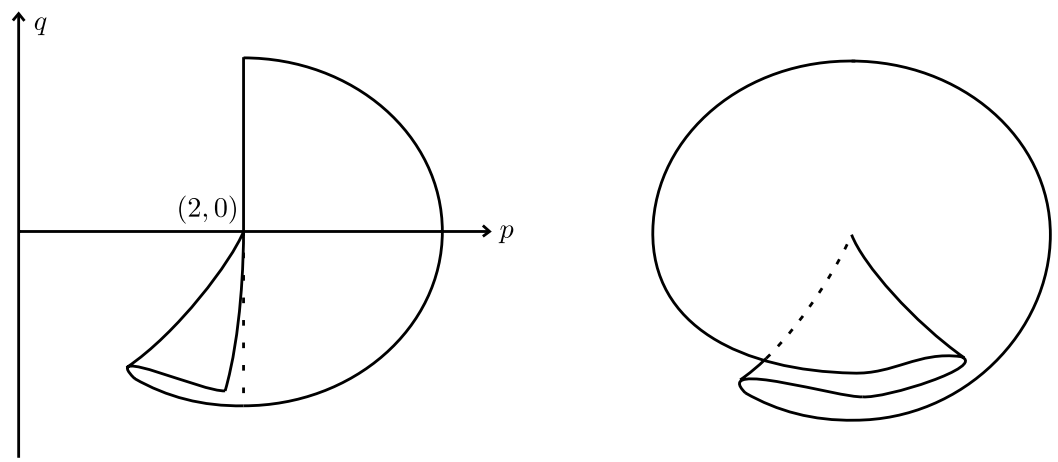

Figure 2: The picture on the right hand side represents a Whitney pleat (a map conjugate to $\left.(x, y) \mapsto\left(x, y^{3}-x y\right)\right)$. The picture on the left hand side shows the situation in Theorem B: half of it.

The image of the folding region is precisely the the curve $p=f(q), q<0$. Thus we have the following addendum to Theorem B:

Addendum to Theorem B Local rigidity fails to hold on the curve $p=f(q)$, $q<0$. In addition, every Dehn filling coefficient in

$$
\{(p, q) \in U \mid f(q)<p<2\}
$$

corresponds to two different spherical structures, and every Dehn filling coefficient $(2, q)$ with $q<0$ corresponds to a spherical structure and a transversely spherical foliation.

By considering straight lines with rational slopes that intersect the curve $p=$ $f(q), q<0$ we obtain the following corollary.

Corollary 1.2 Local rigidity fails to hold for some spherical cone manifolds with singular set a knot and large cone angles.

In 1998 Casson showed that local rigidity fails for some hyperbolic cone manifolds with singular set a graph. Local rigidity for compact hyperbolic cone manifolds with singular set a link and cone angles $\leq 2 \pi$ has been proved by Hodgson and Kerckhoff in [13]. Likely, their methods can be adapted to the situation in the spherical case, but our corollary shows that an upper bound of the cone angle is essential in the spherical case. 
The proof of Theorems A and B allows to prove the the following metric properties of the family of collapsing cone manifolds.

Proposition 1.3 Let $C_{\alpha}$ denote the hyperbolic cone manifolds provided by Theorem A, with $\alpha \in(\pi-\varepsilon, \pi)$, and let $\Sigma_{\alpha}$ denote its singular set. Then:

$$
\lim _{\alpha \rightarrow \pi^{-}} \frac{\operatorname{vol}\left(C_{\alpha}\right)}{(\pi-\alpha) \operatorname{length}\left(\Sigma_{\alpha}\right)}=\frac{3}{8} \quad \text { and } \quad \lim _{\alpha \rightarrow \pi^{-}} \frac{\operatorname{length}\left(\Sigma_{\alpha}\right)}{(\pi-\alpha)^{1 / 3}}=l_{0}>0 .
$$

Proposition 1.4 Let $f$ and $g$ be the functions of Theorem $B$ and let $l_{0}>0$ be as in previous proposition. Then:

$$
\lim _{q \rightarrow 0^{-}} \frac{2-f(q)}{|q|^{3 / 2}}=\lim _{q \rightarrow 0^{-}} \frac{g(q)-2}{|q|^{3 / 2}}=\frac{4}{9 \sqrt[4]{3} \pi} l_{0}^{3 / 2} .
$$

In the proof of Theorem B, we first construct spaces of geometric structures on $M$ parametred by $(s, t) \in U \subset \mathbb{R}^{2}$, where $U$ a neighborhood of the origin (Theorem 3.1). We consider spaces of both, hyperbolic and spherical structures, and work with unified notation: $\mathbb{X}^{3}$ denotes either $\mathbb{H}^{3}$ or $\mathbb{S}^{3}$. Those structures are non degenerate except when $s=0$ or $t=0$. The degenerated structures are the following ones: the origin corresponds to the original Nil structure, the line $t=0$ to Euclidean structures, and the line $s=0$ to transversely hyperbolic or spherical foliations. This space of structures $U$ has symmetry: $( \pm s, \pm t)$ is the parameter of the same structure as $(s, t)$ up to changing the orientation or the spin structure.

Next we construct a deformation space $D e f$, which is a half disc centered at the origin with parameters $(s, \tau)$, with $s \geq 0$, and $\tau=t^{2}$ in the hyperbolic case, $\tau=-t^{2}$ in the spherical case, and $\tau=0$ in the Euclidean one. In the proof of Theorem B, we show that the Dehn filling coefficients $(p, q)$ define an analytic map on $(s, \tau)$ that has "half Whitney pleat" at the origin, as illustrated in Figure 2.

To construct the structures of Theorem 3.1 with parameters $(s, t) \in U$, we need to construct a family of representations $\rho_{(s, t)}$ of $\pi_{1} M$ in $\operatorname{Isom}^{+}\left(\mathbb{X}^{3}\right)$, which are going to be the holonomy representations of the structures. In fact we work in the universal covering of $\operatorname{Isom}^{+}\left(\mathbb{X}^{3}\right)$, that we denote by $G$. When $\mathbb{X}^{3}=\mathbb{H}^{3}$ then $G=S L_{2}(\mathbb{C})$, and when $\mathbb{X}^{3}=\mathbb{S}^{3}$ then $G=S U(2) \times S U(2)$.

The starting point in the construction of $\rho_{(s, t)}$ is the holonomy representation

$$
\text { hol: } \pi_{1} M \rightarrow \operatorname{Isom}(N i l)
$$

and the exact sequences:

$$
0 \rightarrow \mathbb{R} \rightarrow \operatorname{Isom}(N i l) \stackrel{\pi}{\longrightarrow} \operatorname{Isom}\left(\mathbb{R}^{2}\right) \rightarrow 1,
$$




$$
0 \rightarrow \mathbb{R}^{2} \rightarrow \operatorname{Isom}\left(\mathbb{R}^{2}\right) \stackrel{\text { ROT }}{\longrightarrow} O(2) \rightarrow 1 .
$$

The first one comes from the Riemannian fibration $\mathbb{R} \rightarrow N i l \rightarrow \mathbb{R}^{2}$ and the second one is well known. We consider the representation

$$
\phi_{0}=\mathrm{ROT} \circ \pi \circ \mathrm{hol}: \pi_{1} M \rightarrow O(2) \subset S O(3)
$$

and we lift it to $\rho_{0}: \pi_{1} M \rightarrow S U(2) \cong \widetilde{S O(3)}$. We fix $x_{0} \in \mathbb{X}^{3}$ and we view $S U(2)$ as the stabilizer of $x_{0}$ in $G$. We construct $\rho_{(s, t)}$ as a perturbation of $\rho_{0}$. The infinitesimal properties of this perturbation are related to the holonomy representation hol and to sections to the above exact sequences, because by composing hol with those sections we obtain cocycles and cochains.

Organization of the paper We start with a review of Nil geometry and the holonomy representation in Section 2, pointing out its cohomological aspects for relating it later to infinitesimal deformations. In Section 3 we construct the deformation spaces for spherical and hyperbolic structures, assuming the existence of suitable representations $\rho_{(s, t)}$. Those representations are constructed in Section 4, and their infinitesimal properties are studied in Section 5. Section 6 is devoted to Euclidean structures, obtained as degeneration of hyperbolic and spherical ones. In Section 7 we analyze the Dehn filling parameters, achieving the proof of Theorem B. The part of Theorem A not contained in Theorem B is proved in Section 8, together with Propositions 1.3 and 1.4. Section 9 is devoted to an example, where the limit $l_{0}$ of Propositions 1.3 and 1.4 is explicitly computed. Finally Section 10 is devoted to the proof of some technical computations in cohomology.

\section{The holonomy representation}

The usual model for $\mathrm{Nil}$ is the Heisenberg group of matrices of the form

$$
N i l=\left\{\left(\begin{array}{ccc}
1 & x & z \\
0 & 1 & y \\
0 & 0 & 1
\end{array}\right) \mid x, y, z \in \mathbb{R}\right\}
$$

which is canonically identified to $\mathbb{R}^{3}$ by taking coordinates $(x, y, z)$. For our purposes it will be convenient to work with another model. Following [18], we consider $\mathbb{R}^{3}$ with the product:

$$
\left(x_{1}, x_{2}, x_{3}\right)\left(y_{1}, y_{2}, y_{3}\right)=\left(x_{1}+y_{1}, x_{2}+y_{2}, x_{3}+y_{3}+x_{1} y_{2}-x_{2} y_{1}\right)
$$

This is another model for $N i l$. The isomorphism between both models is given by $x=\sqrt{2} x_{1}, y=\sqrt{2} x_{2}$ and $z=x_{3}+x_{1} x_{2}$. 


\subsection{The isometry group of $N i l$}

We consider a 2-parameter family of left-invariant metrics

$$
d s^{2}=\lambda^{2}\left(d x_{1}^{2}+d x_{2}^{2}\right)+\mu^{2}\left(d x_{3}+x_{2} d x_{1}-x_{1} d x_{2}\right)^{2}
$$

for $\lambda, \mu \in \mathbb{R}-\{0\}$. All these metrics have the same 4-dimensional isometry group Isom $(\mathrm{Nil})$. This group $\operatorname{Isom}(\mathrm{Nil})$ preserves the orientation, it has two components, and it is a semi-direct product

$$
\operatorname{Isom}(N i l) \cong N i l \rtimes O(2) .
$$

The group $O(2)$ acts on $N i l$ linearly as the projection of the standard action of $O(2) \subset S O(3)$ on $\mathbb{R}^{3} \cong N i l$ preserving the plane $x_{3}=0$. To see that this action is an isometry, it may be useful to write the metric in cylindrical coordinates $x_{1}=r \cos \theta$ and $x_{2}=r \sin \theta$ :

$$
d s^{2}=\lambda^{2}\left(d r^{2}+r^{2} d \theta^{2}\right)+\mu^{2}\left(d x_{3}-r^{2} d \theta\right)^{2} .
$$

The projection $N i l \rightarrow \mathbb{R}^{2}$ that maps $\left(x_{1}, x_{2}, x_{3}\right) \in N i l$ to $\left(x_{1}, x_{2}\right) \in \mathbb{R}^{2}$ is a Riemannian fibration with fibre a line $\mathbb{R}$. This fibration is preserved by the isometry group and induces an exact sequence

$$
0 \rightarrow \mathbb{R} \rightarrow \operatorname{Isom}(N i l) \stackrel{\pi}{\longrightarrow} \operatorname{Isom}\left(\mathbb{R}^{2}\right) \rightarrow 1 .
$$

A section

$$
\operatorname{VERT}_{p}: \operatorname{Isom}(N i l) \rightarrow \mathbb{R}
$$

may be constructed by fixing a base point $p \in N i l$ as follows: for any $g \in$ $\operatorname{Isom}(N i l), \operatorname{VERT}_{p}(g)$ is the third coordinate of $g(p) p^{-1}$.

On the other hand, we have the well known split exact sequence

$$
0 \rightarrow \mathbb{R}^{2} \rightarrow \operatorname{Isom}\left(\mathbb{R}^{2}\right) \stackrel{\mathrm{ROT}}{\longrightarrow} O(2) \rightarrow 1 .
$$

A section

$$
\operatorname{TRANS}_{q}: \operatorname{Isom}\left(\mathbb{R}^{2}\right) \rightarrow \mathbb{R}^{2}
$$

may also be constructed fixing a base point $q \in \mathbb{R}^{2}$. For any $q \in \operatorname{Isom}\left(\mathbb{R}^{2}\right)$, $\operatorname{TRANS}_{q}(g)=g(q)-q \in \mathbb{R}^{2}$.

\subsection{The holonomy representation}

Our starting point is the holonomy representation of the orbifold $\mathcal{O}$ :

$$
\text { hol: } \pi_{1}^{o}(\mathcal{O}) \rightarrow \operatorname{Isom}(N i l)
$$

and the representation induced on the open manifold $M=|\mathcal{O}|-\Sigma$. 
Definition 2.1 Given the induced representation hol: $\pi_{1} M \rightarrow \operatorname{Isom}(N i l)$, $p \in N i l$ and $q=\pi(p) \in \mathbb{R}^{2}$, we define the following maps:

$$
\begin{aligned}
& \phi_{0}=\mathrm{ROT} \circ \pi \circ \mathrm{hol}: \pi_{1} M \rightarrow O(2) \subset S O(3), \\
& z_{q}=\text { TRANS }_{q} \circ \pi \circ \text { hol: } \pi_{1} M \rightarrow \mathbb{R}^{2} \text {, } \\
& c_{p}=\text { VERT }_{p} \circ \text { hol: } \pi_{1} M \rightarrow \mathbb{R} \text {. }
\end{aligned}
$$

Those three maps determine uniquely the representation hol. It is clear that $\phi_{0}$ is also a representation, but $z_{q}$ and $c_{p}$ are not. However they satisfy some cohomological conditions that we describe next. To do it, we view both $\mathbb{R}^{2}=$ $\mathbb{R}^{2} \times 0$ and $\mathbb{R}=0 \times \mathbb{R}$ as subspaces of $\mathbb{R}^{3}$, therefore they are $\pi_{1} M$-modules via $\phi_{0}: \pi_{1} M \rightarrow O(2) \subset S O(3)$.

The map $z_{q}$ is a cocycle twisted by $\phi_{0}$. This is,

$$
z_{q}\left(g_{1} g_{2}\right)=z_{q}\left(g_{1}\right)+\phi_{0}\left(g_{1}\right) z_{q}\left(g_{2}\right), \quad \forall g_{1}, g_{2} \in \pi_{1} M
$$

The map $c_{p}$ satisfies the following relation:

$$
c_{p}\left(g_{1} g_{2}\right)-c_{p}\left(g_{1}\right)-\phi_{0}\left(g_{1}\right) c_{p}\left(g_{2}\right)=z_{q}\left(g_{1}\right) \times \phi_{0}\left(g_{1}\right) z_{q}\left(g_{2}\right), \quad \forall g_{1}, g_{2} \in \pi_{1} M,
$$

where $\times$ denotes the usual cross product in $\mathbb{R}^{3}$. In cohomology terms, the previous inequality is:

$$
\delta\left(c_{p}\right)=z_{q} \cup z_{q},
$$

where $\delta$ denotes the cobundary, and $\cup$, the cup product associated to $\times$.

The set of all cochains (ie, maps $\pi_{1} M \rightarrow \mathbb{R}^{2} \times 0$ ) is a vector space denoted by $C^{1}\left(\pi_{1} M, \mathbb{R}^{2} \times 0\right)$. The subspace of all cocycles (ie, maps $\pi_{1} M \rightarrow \mathbb{R}^{2} \times 0$ satisfying (3)) is denoted by $Z^{1}\left(\pi_{1} M, \mathbb{R}^{2} \times 0\right)$. Hence $z_{q} \in Z^{1}\left(\pi_{1} M, \mathbb{R}^{2} \times 0\right)$.

Let $B^{1}\left(\pi_{1} M, \mathbb{R}^{2} \times 0\right)$ denote the subspace of all coboundaries, ie, cocycles $b_{r}$ with the property that there exists $r \in \mathbb{R}^{2} \times 0$ with $b_{r}(g)=r-\phi_{0}(g)(r)$, $\forall g \in \pi_{1} M$. The cocycle $z_{q} \notin B^{1}\left(\pi_{1} M, \mathbb{R}^{2} \times 0\right)$ because $z_{q}$ does not have a global fixed point in $\mathbb{R}^{2} \times 0$. Thus the cohomology class of $z_{q}$ in

$$
H^{1}\left(\pi_{1} M, \mathbb{R}^{2} \times 0\right)=Z^{1}\left(\pi_{1} M, \mathbb{R}^{2} \times 0\right) / B^{1}\left(\pi_{1} M, \mathbb{R}^{2} \times 0\right)
$$

is not zero, and it may be easily checked that it is independent of the choice of $q \in \mathbb{R}^{2} \times 0$.

We will prove at the end of the paper that

$$
H^{1}\left(\pi_{1} M, \mathbb{R}^{2} \times 0\right) \cong \mathbb{R} \quad \text { and } \quad H^{1}\left(\pi_{1} M, 0 \times \mathbb{R}\right) \cong 0 .
$$

This has two consequences. Firstly $z_{q}$ is unique up to the choice of $q$ and up to homoteties. Secondly, once $z_{q}$ and $p \in \pi^{-1}(q)$ have been fixed, then $c_{p}$ is unique. 
Different choices of the cohomology class $\left[z_{p}\right]$ correspond to the composition of the holonomy with an automorphism:

$$
\begin{aligned}
N i l & \rightarrow N i l \\
\left(x_{1}, x_{2}, x_{3}\right) & \mapsto\left(\lambda x_{1}, \lambda x_{2}, \lambda^{2} x_{3}\right)
\end{aligned}
$$

for some $\lambda \in \mathbb{R}-\{0\}$.

\subsection{Lifting the holonomy}

We recall that $M=|O|-\Sigma$ and that $\phi_{0}=$ ROT $\circ \pi \circ$ hol $: \pi_{1} \mathcal{O} \rightarrow O(2) \subset S O(3)$. The representation of $\pi_{1} M$ in $S O(3)$ induced by $\phi_{0}$ lifts to a representation to $S U(2) \cong \operatorname{Spin}(3)=\widetilde{S O(3)}$, because we can view it as the holonomy of a non-complete structure on $M$ and apply the following result of Culler [9].

Lemma 2.2 [9] A spin structure on $M$ determines a lift of ROT $\circ \pi \circ$ hol to $\operatorname{Spin}(3) \cong S U(2)$. In particular, since $\operatorname{dim}(M)=3$ there exists a lift.

Remark Two spin structures determine a morphism $\theta: \pi_{1} M \rightarrow \mathbb{Z} / 2 \mathbb{Z}$. It follows from the construction of [9], that if $\rho_{1}$ and $\rho_{2}$ are the lifts associated to these structures, then

$$
\rho_{1}(g)=(-1)^{\theta(g)} \rho_{2}(g) \quad \text { for every } g \in \pi_{1} M .
$$

From now on we fix a spin structure on $M$, hence we also fix a lift of $\phi_{0}=$ ROT $\circ \pi \circ$ hol:

$$
\rho_{0}: \pi_{1} M \rightarrow S U(2)
$$

\subsection{Changing the spin structure}

We consider the natural surjection

$$
\theta: \pi_{1} M \rightarrow \mathbb{Z} / 2 \mathbb{Z}
$$

which is the composition of $\phi_{0}: \pi_{1} M \rightarrow O(2)$ with the projection $O(2) \rightarrow$ $\pi_{0}(O(2)) \cong \mathbb{Z} / 2 \mathbb{Z}$.

We consider the change of spin structure associate to $\theta$.

If $\rho$ is the lift of a representations of $\pi_{1} M$ in $\operatorname{Isom}^{+}\left(\mathbb{X}^{3}\right)$ as in Lemma 2.2, this change of spin structure corresponds to to replace the lift $\rho$ by $(-1)^{\theta} \rho$.

Lemma 2.3 The representation $(-1)^{\theta} \rho_{0}$ is conjugate to $\rho_{0}$. 
Proof It suffices to check that trace $\left((-1)^{\theta} \rho_{0}(g)\right)=\operatorname{trace}\left(\rho_{0}(g)\right)$, for every $g \in \pi_{1} M$, because $\rho_{0}$ is a representation in $S U(2)$. If $g \in \operatorname{ker} \theta$, then the equality of traces holds true because $(-1)^{\theta(g)} \rho_{0}(g)=\rho_{0}(g)$. If $\theta(g)=1$ then $\operatorname{ROT} \circ \pi \circ \operatorname{hol}(g)$ is a rotation of angle $\pi$, as every element in $O(2)-S O(2)$ viewed in $S O(3)$. Hence trace $\left(\rho_{0}(g)\right)=0$ and therefore trace $\left((-1)^{\theta} \rho_{0}(g)\right)=$ $-\operatorname{trace}\left(\rho_{0}(g)\right)=0$.

\section{Deformation spaces}

From now on $\mathbb{X}^{3}$ will denote $\mathbb{H}^{3}$ and $\mathbb{S}^{3}$. Every statement about $\mathbb{X}^{3}$ will be understood to be a statement about both, the hyperbolic space and the 3 sphere. The hyperbolic plane and the 2 -sphere will be denoted by $\mathbb{X}^{2}$.

\subsection{Spaces of geometric structures}

Theorem 3.1 There exists a space of geometric structures on $M=\mathcal{O}-\Sigma$ with Dehn filling end parametrized by a neighborhood of the origin $U \subset \mathbb{R}^{2}$. According to the parameters $(s, t) \in U$, the structure is of the following kind:

(i) the original Nil structure, when $(s, t)=0$;

(ii) modeled on $\mathbb{X}^{3}$, when $s t \neq 0$;

(iii) a foliation transversely modelled on $\mathbb{X}^{2}$, when $s=0, t \neq 0$; and

(iv) a Euclidean structure, when $s \neq 0, t=0$.

In addition, those structures are oriented and equipped with a spin structure, so that $(s,-t)$ and $(s, t)$ correspond to structures with opposite orientation, and $-(s, t)$ and $(s, t)$ correspond to the spin structures differing by $\theta$.

A Dehn filling end for the structure on $T^{2} \times(0,1]$ means the following. There is a geodesic $\gamma \subset \mathbb{X}^{3}$ such that the developing map $D: \widetilde{T^{2}} \times(0,1] \rightarrow \mathbb{X}^{3}$ maps $\{x\} \times(0,1]$ to a minimizing segment between $D(x, 1)$ and $\gamma$, for every $x \in \widetilde{T^{2}}$. In addition, the parameter in $(0,1]$ is proportional to arc-length.

The parameter $(s, t)$ has the following interpretation. We choose $l, m \in \pi_{1} M$ so that they generate a peripheral group and $m$ is a meridian for $\Sigma$. We may choose $l$ so that $\phi_{0}(l)$ is trivial. The rotation angle and the translation length of the holonomy of $l$ are respectively $s$ and $t$. 
Convention We fix $x_{0} \in \mathbb{X}^{3}$ and we view $\phi_{0}$ as a representation in $\operatorname{Isom}^{+}\left(\mathbb{X}^{3}\right)$ that fixes $x_{0}$, because $S O(3)$ is the stabilizer of a point in $\operatorname{Isom}^{+}\left(\mathbb{X}^{3}\right)$. We also fix $\left\{e_{1}, e_{2}, e_{3}\right\}$ a positive orthonormal basis for $\mathbb{R}^{3}$ so that $\left\langle e_{1}, e_{2}\right\rangle=\mathbb{R}^{2} \times 0$ and $\left\langle e_{3}\right\rangle=0 \times \mathbb{R}$ are the subspaces invariant by $O(2)$. The totally geodesic plane tangent to $\mathbb{R}^{2} \times 0 \subset T_{x_{0}} \mathbb{X}^{3}$ is denoted by $\mathbb{X}^{2}=\exp _{x_{0}}\left(\mathbb{R}^{2} \times 0\right)$.

The following maps from Nil to $\mathbb{X}^{3}$ will be used in the proof of Theorem 3.1.

Definition 3.2 For $(s, t) \in \mathbb{R}^{2}$ we define:

$$
\begin{aligned}
\Delta_{(s, t)}: N i l \cong \mathbb{R}^{3} & \rightarrow \mathbb{X}^{3} \\
\left(x_{1}, x_{2}, x_{3}\right) & \mapsto \exp _{x_{0}}\left(t\left(x_{1} e_{1}+x_{2} e_{2}+s x_{3} e_{3}\right)\right)
\end{aligned}
$$

where $\exp _{x_{0}}$ denotes the Riemannian exponential at the point $x_{0} \in \mathbb{X}^{3}$. Here we have identified $N i l$ with $\mathbb{R}^{3}$.

Notice that, when $s t \neq 0, \Delta_{(s, t)}$ is a local diffeomorphism, and when $s=0$ but $t \neq 0$, it is a local a submersion of rank 2 onto $\mathbb{X}^{2}$.

\subsection{Deformations of representations}

Proposition 3.3 There exists a perturbation $\rho_{(s, t)}: \pi_{1} M \rightarrow G$ of $\rho_{0}$, with parameter $(s, t) \in U \subset \mathbb{R}^{2}$, such that:

(i) $\rho_{(s, 0)}$ stabilizes $x_{0}$.

(ii) $\rho_{(0, t)}$ stabilizes $\mathbb{X}^{2}=\exp _{x_{0}}\left(\mathbb{R}^{2} \times 0\right)$

(iii) For every $g \in \pi_{1} M$

$$
\lim _{\substack{(s, t) \rightarrow 0 \\ s t \neq 0}} \Delta_{(s, t)}^{-1} \circ \rho_{(s, t)}(g) \circ \Delta_{(s, t)}=\operatorname{hol}(g)
$$

uniformly on compact subsets of $N$ il for the $\mathcal{C}^{1}$-topology.

(iv) Let $\bar{\Delta}_{t}=\left.\Delta_{(0, t)}\right|_{\mathbb{R}^{2} \times 0}$. For every $g \in \pi_{1} M$

$$
\lim _{t \rightarrow 0} \bar{\Delta}_{t}^{-1} \circ \rho_{(0, t)}(g) \circ \bar{\Delta}_{(0, t)}=\pi \circ \operatorname{hol}(g)
$$

uniformly on compact subsets of $\mathbb{R}^{2} \times 0$ for the $\mathcal{C}^{1}$-topology.

(v) The representations $\rho_{(-s,-t)}$ and $(-1)^{\theta} \rho_{(s, t)}$ are conjugate in $\widetilde{\operatorname{Isom}^{+}}\left(\mathbb{X}^{3}\right)$.

(vi) $\rho_{(-s, t)}$ and $\rho_{(s, t)}$ are conjugate by an orientation reversing element in $\widetilde{\operatorname{Isom}}\left(\mathbb{X}^{3}\right)$. 
We shall prove Theorem 3.1 assuming this proposition. The perturbation we will construct satisfy some more properties related to the Euclidean structures, when $t=0$. These properties will be explained later, hence for the moment we will not prove the part of Theorem 3.1 concerning Euclidean structures.

Properties (iii) and (iv) of the proposition are related to the infinitesimal properties of $\rho_{(s, t)}$ and to the cocycle $z_{q}$ and the cochain $c_{q}$.

\subsection{Proof of Theorem 3.1}

We construct a covering $\left\{U_{i}\right\}_{i=0, \ldots, n}$ of $M$ such that $U_{i}$ is 1 -connected for $i \geq 1$ and $U_{0}$ is a neighborhood of the end of $M$.

Since $U_{1}$ is simply connected, the lift of $U_{1}$ in the universal covering of $M$ is

$$
\widetilde{U_{1}}=\bigcup_{g \in \pi_{1} M} g W_{1}
$$

for some open set $W_{1} \subset \widetilde{M}$ that projects homeomorphically to $U_{1}$. We define on $W_{1}$

$$
\left.D_{(s, t)}\right|_{W_{1}}=\left.\Delta_{(s, t)} \circ \mathcal{D}_{0}\right|_{W_{1}}: W_{1} \rightarrow \mathbb{X}^{3}
$$

Where $\mathcal{D}_{0}: \widetilde{M} \rightarrow N i l$ is the holonomy for the Nil structure. Next we define $D_{(s, t)}$ on $\widetilde{U_{1}}$ by taking the equivariant extension. By Proposition 3.3 (iii),

$$
\left.\lim _{\substack{(s, t) \rightarrow 0 \\ s t \neq 0}} \Delta_{(s, t)}^{-1} \circ D_{(s, t)}\right|_{\widetilde{U_{1}}}=\left.\mathcal{D}_{0}\right|_{\widetilde{U_{1}}}
$$

for the $\mathcal{C}^{1}$-topology uniformly on compact subsets.

We make the same construction for all sets $U_{i}$ with $i \geq 2$ and for $U_{0}$ we make a cylindrical construction taking care of the holonomy at the end. We glue the final construction by using standard techniques about bump functions and refinements, as explained in [7] (and also in [17, 11]), so we obtain a family $D_{(s, t)}$ of maps that are $\rho_{(s, t)}$-equivariant and such that:

$$
\lim _{\substack{(s, t) \rightarrow 0 \\ s t \neq 0}} \Delta_{(s, t)}^{-1} \circ D_{(s, t)}=\mathcal{D}_{0}
$$

for the $\mathcal{C}^{1}$-topology uniformly on compact subsets. In particular $D_{(s, t)}$ is a local diffeo for small values of $(s, t)$ with $s t \neq 0$.

For the neighborhood $U_{0}$ we need to be more careful. Let $\gamma=\exp _{x_{0}}\left\langle e_{1}\right\rangle$ be the geodesic preserved by $\rho_{0}(m)$. We will construct $\rho_{(s, t)}$ so that $\gamma$ is 
preserved by $\rho_{(s, t)}(m)$ (and also by $\rho_{(s, t)}(l)$, by commutativity). It will also follow from the construction that $\rho_{(s, t)}(l)$ is the composition of a translation of length $t$ with a rotation of angle $s$ around $\gamma$. We consider the family of maps $\Theta_{(s, t)}: \tilde{U}_{0} \rightarrow \mathbb{R}^{3}-\left\langle e_{1}\right\rangle$ such that $\Theta_{0}$ is the developing map $\mathcal{D}_{0}$ restricted to $\tilde{U}_{0}$, the distance from $\Theta_{(s, t)}(x)$ to $\left\langle e_{1}\right\rangle$ is independent of $(s, t)$ and $\Theta$ is $\pi_{1} U_{0}-$ equivariant by the action of $\rho_{(s, t)}$. Then we define $\left.\mathcal{D}_{(s, t)}\right|_{\tilde{U}_{0}}=\Delta_{(s, t)} \circ \Theta_{(s, t)}$ and we glue it in the same way.

This proves assertion (ii) of Theorem 3.1. The proof of assertion (iii) is quite similar by using Proposition 3.3. The properties about symmetries are also clear from Proposition 3.3.

We recall that the part of the theorem concerning Euclidean structures will be proved later.

\section{Construction of the representations}

In this section we construct the representations of Proposition 3.3.

\subsection{Smoothness of the varieties of characters}

We work with the varieties of representations of $\pi_{1} M$ in $S U(2)$ and $S L_{2}(\mathbb{C})$ :

$$
\begin{aligned}
R(M, S U(2)) & =\operatorname{Hom}\left(\pi_{1} M, S U(2)\right), \\
R\left(M, S L_{2}(\mathbb{C})\right) & =\operatorname{Hom}\left(\pi_{1} M, S L_{2}(\mathbb{C})\right) .
\end{aligned}
$$

The varieties of characters are defined as:

$$
\begin{aligned}
X(M, S U(2)) & =R(M, S U(2)) / S U(2), \\
X\left(M, S L_{2}(\mathbb{C})\right) & =R\left(M, S L_{2}(\mathbb{C})\right) / / S L_{2}(\mathbb{C}) .
\end{aligned}
$$

The symbol // in the definition of $X\left(M, S L_{2}(\mathbb{C})\right)$ means the algebraic quotient (in invariant theory). In particular $X\left(M, S L_{2}(\mathbb{C})\right.$ ) is algebraic affine (also defined over $\mathbb{Q})$. However since $S U(2)$ is compact but not complex, $X(M, S U(2))$ is just the topological quotient, and it is only real semi-algebraic, contained in the set of real points of $X\left(M, S L_{2}(\mathbb{C})\right)$.

Every point in $X\left(M, S L_{2}(\mathbb{C})\right)$ is the character of a representation in $S L_{2}(\mathbb{C})$, ie, a map

$$
\begin{aligned}
\chi_{\rho}: \pi_{1} M & \rightarrow \mathbb{C} \\
\gamma & \mapsto \operatorname{trace}(\rho(\gamma))
\end{aligned}
$$


for some $\rho \in R\left(M, S L_{2}(\mathbb{C})\right)$. Every conjugacy class of representation into $S U(2)$ is determined by its character, therefore the notation makes sense and $X(M, S U(2)) \subset X\left(M, S L_{2}(\mathbb{C})\right)$.

Definition 4.1 For every $\gamma \in \pi_{1} M, I_{\gamma}: X\left(M, S L_{2}(\mathbb{C})\right) \rightarrow \mathbb{C}$ denotes the evaluation map. In other words, it is the map induced by the trace function:

$$
I_{\gamma}\left(\chi_{\rho}\right)=\chi_{\rho}(\gamma)=\operatorname{trace}(\rho(\gamma)) .
$$

Proposition 4.2 The character $\chi_{0}$ of $\rho_{0}$ is a smooth one dimensional point of both $X(M, S U(2))$ and $X\left(M, S L_{2}(\mathbb{C})\right)$.

Proof We first prove the proposition for $X\left(M, S L_{2}(\mathbb{C})\right)$. By a Theorem 5.6 of Thurston's notes [20], the local dimension of $X\left(M, S L_{2}(\mathbb{C})\right)$ at the character of $\rho_{0}$ is at least one. It suffices to prove that $H^{1}\left(\pi_{1} M, s l_{2}(\mathbb{C})\right) \cong \mathbb{C}$, (where $\pi_{1} M$ acts on $s l_{2}(\mathbb{C})$ via $\left.A d \rho_{0}\right)$ because this cohomology group contains the Zariski tangent space of $X\left(M, S L_{2}(\mathbb{C})\right)$ at $\chi_{0}$. We have said before that $H^{1}\left(\pi_{1} M, \mathbb{R}^{2} \times\right.$ $0) \cong \mathbb{R}$ and $H^{1}\left(\pi_{1} M, 0 \times \mathbb{R}\right) \cong 0$. Therefore

$$
H^{1}\left(\pi_{1} M, s u(2)\right) \cong H^{1}\left(\pi_{1} M, \mathbb{R}^{3}\right) \cong \mathbb{R},
$$

because $s u(2)$ and $\mathbb{R}^{3}$ are isomorphic as $\pi_{1} M$-modules. In particular

$$
H^{1}\left(\pi_{1} M, s l_{2}(\mathbb{C})\right) \cong H^{1}\left(\pi_{1} M, s u(2)\right) \otimes_{\mathbb{R}} \mathbb{C} \cong \mathbb{C} .
$$

The proposition for $X(M, S U(2))$ follows easily, using the fact that the variety $X\left(M, S L_{2}(\mathbb{C})\right)$ is defined over $\mathbb{R}$ and a neighborhood of $\chi_{0}$ in $X\left(M, S L_{2}(\mathbb{C})\right) \cap$ $\mathbb{R}^{N}$ coincides with $X(M, S U(2))$.

\subsection{Local parametrization}

We construct a local parameter of a neighborhood of $\chi_{0}$ in $X\left(M, S L_{2}(\mathbb{C})\right)$. We choose $l, m \in \pi_{1} M$ so that they generate a peripheral subgroup $\pi_{1} T^{2}$. We assume that $m$ is a meridian of $\Sigma$. We also assume that $\theta(l)=0$, by replacing $l$ by $l m$ if necessary.

Remark We have that $\rho_{0}(l)= \pm I d$, because $l$ and $m$ commute, and $l \in \operatorname{ker} \theta$ but $\theta(m)=1$ (ie, $\phi_{0}(l) \in S O(2)$ but $\left.\phi_{0}(m) \in O(2)-S O(2)\right)$.

The idea is to choose $w=\alpha_{l}$ the angle rotation of $\rho(l)$ as a local parameter of $X(M, S U(2))$ (so that its extension to $X\left(M, S L_{2}(\mathbb{C})\right.$ ) corresponds to $\pm 2 i$ times the logarithm of an eigenvalue). The sign of this angle is determined by the 
sense of rotation around the invariant geodesic, which corresponds to a choice of the spin structure and determines the choice of the lift $\rho_{0}$. We would like to define $w$ as $2 \arccos \left(I_{l} / 2\right)$, but arccos is not well defined in a neighborhood of \pm 1 . Formally, we can define it as follows.

Definition 4.3 In a neighborhood of $\chi_{0}$ we define $w$ as

$$
w=2 \arccos \left(I_{l m} / 2\right)-2 \arccos \left(I_{m} / 2\right) .
$$

so that $I_{l}= \pm 2 \cos \frac{w}{2}$.

Lemma 4.4 The function $w$ defines a local parametrization of both varieties of characters $X(M, S U(2))$ and $X\left(M, S L_{2}(\mathbb{C})\right)$.

Proof It follows from the proof of Proposition 4.2 that $H^{1}\left(\pi_{1} M, s u(2)\right)$ is isomorphic to the tangent space $T_{\chi_{0}} X(M, S U(2))$. Thus we view $H_{1}\left(\pi_{1} M\right.$, $s u(2))$ as the cotangent space $T_{\chi_{0}}^{1} X(M, S U(2)) \cong \mathbb{R}$, and it is sufficient to check that the differential form $d w \neq 0$. In particular, we just need to prove that the Kronecker pairing $\left\langle d w, z_{q}\right\rangle$ does not vanish, where $z_{q}$ is the cocycle defined in Subsection 2.2. Since, for a representation $\rho, w\left(\chi_{\rho}\right)$ is precisely the angle of $\rho(l)$, Proposition 9.6 in [16] implies that $\left\langle d w, z_{q}\right\rangle$ is precisely the translation length of $\pi \circ \operatorname{hol}(l)$. This length is non-zero because $\Sigma$ is horizontal.

We recall that $\theta: \pi_{1} M \rightarrow \mathbb{Z} / 2 \mathbb{Z}$ is the composition of $\phi_{0}: \pi_{1} M \rightarrow O(2)$ with the projection $O(2) \rightarrow \pi_{0}(O(2)) \cong \mathbb{Z} / 2 \mathbb{Z}$. We consider the change of spin structure associate to $\theta$. For a representation $\rho \in R\left(M, S L_{2}(\mathbb{C})\right)$, to change the spin structure corresponds to replace $\rho$ by $(-1)^{\theta} \rho$.

Lemma $4.5 w\left(\chi_{(-1)^{\theta} \rho}\right)=-w\left(\chi_{\rho}\right)$.

Proof Since $\chi_{0}$ is invariant by $\theta$ (Lemma 2.3), the neighborhood of $\chi_{0}$ may be chosen invariant by the change of the spin structure. Since $\theta(m)=\theta(l m)=1$, we have that $I_{m}\left(\chi_{(-1)^{\theta} \rho}\right)=-I_{m}\left(\chi_{\rho}\right)$ and $I_{l m}\left(\chi_{(-1)^{\theta} \rho}\right)=-I_{l m}\left(\chi_{\rho}\right)$. Therefore, for the branch of $\arccos$ with $\arccos (0)=\pi / 2$, we have:

$$
\begin{aligned}
2 \arccos \left(-I_{m}(\chi) / 2\right) & =\pi-2 \arccos \left(I_{m}(\chi) / 2\right) \\
2 \arccos \left(-I_{l m}(\chi) / 2\right) & =\pi-2 \arccos \left(I_{l m}(\chi) / 2\right)
\end{aligned}
$$

and the lemma follows. 


\subsection{Deformations of characters}

We choose different varieties of characters for the hyperbolic and the spherical case, but we will unify the notation for the neighborhood $U$.

In the hyperbolic case, since $\operatorname{Isom}^{+}\left(\mathbb{H}^{3}\right) \cong P S L_{2}(\mathbb{C})$ we work in $X\left(M, S L_{2}(\mathbb{C})\right)$ (we recall that we have fixed a spin structure, hence all holonomy representations have a natural lift). We fix $U \subset \mathbb{R}^{2}$ a neighborhood of the origin, with coordinates $(s, t) \in U$ and set

$$
w=s-t i,
$$

so that, for any representation $\varrho_{w}$ with character $w$, the complex length of $\varrho_{w}(l)$ is $i w=t+s i$ (ie, a translation of length $t$ plus a rotation of angle $s$ ).

In the spherical case, since $\operatorname{Spin}(4) \cong S U(2) \times S U(2)$ we work in $X(M, S U(2)) \times$ $X(M, S U(2))$. We denote by $w_{1}$ and $w_{2}$ the ordered (real) parameteters of each factor $X(M, S U(2))$ given by Definition 4.3. We fix $U \subset \mathbb{R}^{2}$ a neighborhood of the origin, with coordinates $(s, t) \in U$ and we set

$$
\left(w_{1}, w_{2}\right)=(s+t, s-t)
$$

Again, any representation with character $\left(w_{1}, w_{2}\right)$ evaluated at $l$ is a translation of length $t$ composed with a rotation of angle $s$ around the same edge.

In both cases, $\chi_{0}$ the character of $\rho_{0}$ has coordinates $(s, t)=(0,0)$. To construct the representations $\rho_{(s, t)}$ we need a section to the projection

$$
R\left(M, S L_{2}(\mathbb{C})\right) \rightarrow X\left(M, S L_{2}(\mathbb{C})\right) .
$$

This will be done after the description of $\rho_{0}$.

\subsection{Description of $\rho_{0}$}

We recall that we have fixed $\left\{e_{1}, e_{2}, e_{3}\right\}$ an orthonormal basis for $\mathbb{R}^{3}$, so that $\left\langle e_{1}, e_{2}\right\rangle=\mathbb{R}^{2} \times 0$ and $\left\langle e_{3}\right\rangle=0 \times \mathbb{R}$ are the subspaces invariant by $\rho_{0}$.

By using the natural identification $s u(2) \cong \mathbb{R}^{3}$ as $S U(2)$-modules, we view $e_{1}, e_{2}, e_{3}$ as three matrices of $s u(2)$ such that the following formula and its cyclic permutations hold:

$$
\left[e_{1}, e_{2}\right]=e_{3},
$$

because the Lie bracket in $s u(2)$ corresponds to the cross product in $\mathbb{R}^{3}$.

Remark Let $v \in s u(2) \cong \mathbb{R}^{3}$ be a unitary vector and $\alpha \in \mathbb{R}$. Then $\exp (\alpha v) \in$ $S U(2)$ projects in $S O(3)$ to a rotation of angle $\alpha$ around $\langle v\rangle$. 
Thus, if $\theta(g)=0$ then $\rho_{0}(g)=\exp \left(\alpha_{g} e_{3}\right)$, for some $\alpha_{g} \in \mathbb{R}$. Notice that

$$
\exp \left(\left(\alpha_{g}+2 \pi\right) e_{3}\right)=-\exp \left(\alpha_{g} e_{3}\right) .
$$

We may also assume that $e_{1}$ is the vector invariant for the meridian $m$ and that the spin structure has been chosen so that $\rho_{0}(m)=\exp \left(\pi e_{1}\right)$. The elements which are not in the kernel of $\theta$ are of the form $g m$ for some $g \in \operatorname{ker}(\theta)$, and we have $\rho_{0}(g m)=\exp \left(\pi\left(\cos \left(\alpha_{g} / 2\right) e_{1}+\sin \left(\alpha_{g} / 2\right) e_{2}\right)\right)$.

Remark The conjugation matrix between $\rho_{0}$ and $(-1)^{\theta} \rho_{0}$ is $\pm \exp \left(\pi e_{3}\right)$.

This remark follows from the description of $\rho_{0}$ and the fact the adjoint action (equivalent to the orthogonal action on $\mathbb{R}^{3}$ ) of $\exp \left(\pi e_{3}\right)$ changes the sign of $e_{1}$ and $e_{2}$ and preserves $e_{3}$.

\subsection{The section for $R\left(M, S L_{2}(\mathbb{C})\right)$}

Lemma 4.6 There exists a neigborhood $V \subset X\left(M, S L_{2}(\mathbb{C})\right)$ and a section $\sigma: V \rightarrow R\left(M, S L_{2}(\mathbb{C})\right)$ such that, if $\varrho_{w}=\sigma(w)$, then $\forall g \in \pi_{1} M$,

$$
\varrho_{w}(g)=\exp \left(f_{g}(w)+h_{g}(w)\right) \rho_{0}(g)
$$

where $f_{g}$ and $h_{g}$ are analytic maps with real coefficients valued on the Lie algebra $s l_{2}(\mathbb{C})$, such that $f_{g}(w) \in\left\langle e_{1}, e_{2}\right\rangle_{\mathbb{C}}, h_{g}(w) \in\left\langle e_{3}\right\rangle_{\mathbb{C}}, f_{g}$ is odd and $h_{g}$ is even.

When we say that the coefficients of $f_{g}$ and $h_{g}$ are real, we mean that for $w \in \mathbb{R}, f_{g}(w), h_{g}(w) \in s u(2)$.

Proof The proof is based in a construction analogue to Luna's slice theorem. We consider the involution $\nu$ on $R\left(M, S L_{2}(\mathbb{C})\right)$ and $R(M, S U(2))$ defined as follows:

$$
\nu(\rho)=(-1)^{\theta} A d_{\exp \left(\pi e_{3}\right)} \circ \rho
$$

where $\theta: \pi_{1} M \rightarrow \mathbb{Z} / 2 \mathbb{Z}$ is described above.

By the remark in Subsection 4.4, $\nu\left(\rho_{0}\right)=\rho_{0}$. In addition, by Lemma 4.5, if $t: R\left(M, S L_{2}(\mathbb{C})\right) \rightarrow X\left(M, S L_{2}(\mathbb{C})\right)$ denotes the projection, then

$$
w \circ t \circ \nu=-w \circ t \text {. }
$$

Lemma 4.7 There exists an algebraic complex curve $\mathcal{S} \subset R\left(M, S L_{2}(\mathbb{C})\right)$ with the following properties: 
(i) $\rho_{0}$ is a smooth point of $\mathcal{S}$.

(ii) The projection $t: R\left(M, S L_{2}(\mathbb{C})\right) \rightarrow X\left(M, S L_{2}(\mathbb{C})\right)$ restricts to a map $\left.t\right|_{\mathcal{S}}: \mathcal{S} \rightarrow X\left(M, S L_{2}(\mathbb{C})\right)$ locally bianalytic at $\rho_{0}$.

(iii) $\mathcal{S}^{\prime}=\mathcal{S} \cap R(M, S U(2))$ is a real curve smooth at $\rho_{0}$ and the restriction $\left.t\right|_{\mathcal{S}^{\prime}}: \mathcal{S}^{\prime} \rightarrow X(M, S U(2))$ is also locally bianalytic at $\rho_{0}$.

(iv) $\mathcal{S}$ is invariant by the involution $\nu$.

(v) For every $\rho \in \mathcal{S}, \rho(m)=\exp \left(\alpha_{\rho} e_{1}\right)$, for some $\alpha_{\rho} \in \mathbb{R}$.

We postpone its proof. Assuming it holds, we conclude the proof of Lemma 4.6. It suffices to take $\sigma=\left.t\right|_{\mathcal{S}} ^{-1}$. We write $\varrho_{w}=\sigma(w)$ and $\varrho_{w}(g)=\exp \left(f_{g}(w)+\right.$ $\left.h_{g}(w)\right) \rho_{0}(g)$ for some analytic maps such that the image of $f_{g}$ is contained in $\left\langle e_{1}, e_{2}\right\rangle_{\mathbb{C}}$ and the image of $h_{g}$ is contained in $\left\langle e_{3}\right\rangle_{\mathbb{C}}$. These maps have real coefficients by assertion (iii) of Lemma 4.7. We use the involution to prove that $f_{g}$ is odd and $h_{g}$ is even. The representations $\varrho_{-w}$ and $\nu\left(\varrho_{w}\right)$ have the same character. By the properties of $\mathcal{S}$, it follows that $\varrho_{-w}=\nu\left(\varrho_{w}\right)$. In addition

$$
\begin{aligned}
\nu\left(\varrho_{w}\right)(g) & =(-1)^{\theta(g)} A d_{\exp \left(\pi e_{3}\right)}\left(\varrho_{w}(g)\right) \\
& =(-1)^{\theta(g)} A d_{\exp \left(\pi e_{3}\right)}\left(\exp \left(f_{g}(w)+h_{g}(w)\right)\right) A d_{\exp \left(\pi e_{3}\right)}\left(\rho_{0}(g)\right) \\
& =\exp \left(-f_{g}(w)+h_{g}(w)\right) \rho_{0}(g)
\end{aligned}
$$

because $(-1)^{\theta(g)} A d_{\exp \left(\pi e_{3}\right)}\left(\rho_{0}(g)\right)=\rho_{0}(g)$ and $A d_{\exp \left(\pi e_{3}\right)}$ changes the sign of $e_{1}$ and $e_{2}$ but preserves $e_{3}$. Comparing equality (4) with

$$
\nu\left(\varrho_{w}\right)(g)=\varrho_{-w}(g)=\exp \left(f_{g}(-w)+h_{g}(-w)\right) \rho_{0}(g)
$$

it follows that $f_{g}$ is odd and $h_{g}$ even, as claimed.

Proof of Lemma 4.7 We choose an element $g_{0} \in \operatorname{ker}(\theta)$ such that $\rho_{0}\left(g_{0}\right)=$ $\exp \left(\alpha_{0} e_{3}\right)$, for some $\alpha_{0} \in \mathbb{R}-2 \pi \mathbb{Z}$. We define:

$$
\mathcal{S}=\left\{\begin{array}{l|c}
\rho \in R\left(M, S L_{2}(\mathbb{C})\right) & \rho(m)=\exp \left(\alpha e_{1}\right), \rho\left(g_{0}\right)=\exp \left(\beta_{1} e_{1}+\beta_{3} e_{3}\right), \\
\text { with } \alpha, \beta_{1}, \beta_{3} \in \mathbb{C}
\end{array}\right\}
$$

The projection $t: R\left(M, S L_{2}(\mathbb{C})\right) \rightarrow X\left(M, S L_{2}(\mathbb{C})\right)$ restricts to a map $\left.t\right|_{\mathcal{S}}: \mathcal{S} \rightarrow$ $X\left(M, S L_{2}(\mathbb{C})\right)$.

Let $e_{0}$ denote the identity matrix of size $2 \times 2$, so that $\left\{e_{0}, e_{1}, e_{2}, e_{3}\right\}$ is a basis for $M_{2}(\mathbb{C})$ as $\mathbb{C}$-vector space. For every $\rho \in R\left(M, S L_{2}(\mathbb{C})\right)$ and every $g \in \pi_{1} M$ we write:

$$
\rho(g)=\sum_{i=0}^{3} \pi_{i, g}(\rho) e_{i} .
$$


If we define $F: R\left(M, S L_{2}(\mathbb{C})\right) \rightarrow \mathbb{C}^{3}$ as $F=\left(\pi_{2, m}, \pi_{3, m}, \pi_{2, g_{0}}\right)$, then $\mathcal{S}=$ $F^{-1}(0)$. An easy computation shows that the differential of $F$ at $\rho_{0}$ maps $B^{1}\left(M, s l_{2}(\mathbb{C})\right)$ isomorphically onto $\mathbb{C}^{3}$. It follows that $\rho_{0}$ is a smooth point of $\mathcal{S}$ and that $\left.t\right|_{\mathcal{S}}$ is locally bianalytic. This proves assertions (i) and (ii) of the proposition.

If in the construction of $\mathcal{S}$ we replace $S L_{2}(\mathbb{C})$ by $S U(2)$, then we obtain $\mathcal{S}^{\prime}$ and the same construction as above applies to prove assertion (iii) of the proposition. Finally assertions (iv) and (v) follow from construction.

\subsection{Sections for the deformation spaces}

Definition 4.8 For $(s, t) \in U$, we define $\left.\rho_{(s, t)} \in R(M, G)\right)$ as follows:

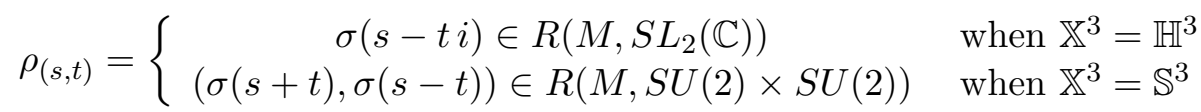

Proposition 4.9 For every $(s, 0) \in U, \rho_{(s, 0)}$ stabilizes $x_{0} \in \mathbb{X}^{3}$, the point stabilized by $\rho_{0}$.

Proof Let $f_{g}$ and $h_{g}$ be the functions of Lemma 4.6. In the hyperbolic case, the proposition follows from the fact that the functions $f_{g}$ and $h_{g}$ have real coefficients: when $t=0, f_{g}(s), h_{g}(s) \in s u(2)$, hence $\rho_{(s, 0)} \in R(M, S U(2))$, and $S U(2)$ is precisely the stabilizer of $x_{0}$. In the spherical case, $\rho_{(s, 0)}$ is diagonal by construction, and the diagonal is precisely the stabilizer of $x_{0}$.

\section{Infinitesimal deformations}

\subsection{Infinitesimal isometries}

Recall that in the convention after Theorem 3.1, we have fixed a point $x_{0}$ so that $\phi_{0}=\mathrm{ROT} \circ \pi \circ \mathrm{hol}$ is a representation into

$$
S O(3) \cong \operatorname{Isom}^{+}\left(X^{3}\right)_{x_{0}} \hookrightarrow \operatorname{Isom}^{+}\left(X^{3}\right) .
$$

Its lift to $S U(2) \cong G_{x_{0}}$ is $\rho_{0}$. Let $\mathbf{g}$ denote the lie algebra of $\operatorname{Isom}^{+}\left(\mathbb{X}^{3}\right)$ and $\mathbf{g}_{x_{0}}$ the Lie subalgebra corresponding to $\operatorname{Isom}^{+}\left(\mathbb{X}^{3}\right)_{x_{0}}$. We have a natural exact sequence

$$
0 \rightarrow \mathbf{g}_{x_{0}} \rightarrow \mathbf{g} \rightarrow T_{x_{0}} \mathbb{X}^{3} \rightarrow 0
$$


The Killing form on $\mathbf{g}$ is non-degenerate, and $T_{x_{0}} \mathbb{X}^{3}$ is naturally identified to the orthogonal space to $\mathbf{g}_{x_{0}}$. We have an orthogonal sum:

$$
\mathbf{g}=\mathbf{g}_{x_{0}} \perp T_{x_{0}} \mathbb{X}^{3}
$$

Definition 5.1 Elements of $\mathrm{g}$ are called infinitesimal isometries; elements of $\mathbf{g}_{x_{0}}$, infinitesimal rotations (with respect to $x_{0}$ ); and elements of $T_{x_{0}} \mathbb{X}^{3}$, infinitesimal translations (with respect to $x_{0}$ ).

Lemma 5.2 There is a natural identification of $S O(3)$-modules:

$$
T_{x_{0}} \mathbb{X}^{3} \cong \mathbb{R}^{3} \cong \mathbf{g}_{x_{0}},
$$

where the action of $S O(3)$ on $\mathbf{g}_{x_{0}}$ and $T_{x_{0}} \mathbb{X}^{3}$ is the adjoint action and the action on $\mathbb{R}^{3}$ is standard. In addition, it preserves the products (cross product on $\mathbb{R}^{3} \cong T_{x_{0}} \mathbb{X}^{3}$ and Lie bracket on $\mathbf{g}_{x_{0}}$ ) and the natural bilinear forms (Killing form on $\mathbf{g}_{x_{0}}$ and the metric on $\mathbb{R}^{3} \cong T_{x_{0}} \mathbb{X}^{3}$ ) up to a constant.

The isomorphism from $T_{x_{0}} \mathbb{X}^{3}$ to $\mathbf{g}_{x_{0}}$ maps the infinitesimal translation of tangent vector $v \in T_{x_{0}} \mathbb{X}^{3}$ to the infinitesimal rotation around the line $\mathbb{R} v$ of infinitesimal angle $|v|$.

It is convenient to specify Lemma 5.2 and isomorphism (5) in the hyperbolic and the spherical case:

(a) In the hyperbolic case $\mathbf{g} \cong s l_{2}(\mathbb{C})$ and $\mathbf{g}_{x_{0}}$ is a subalgebra conjugate to $s u(2)$. In this case, isomorphism (5) is written as:

$$
s l_{2}(\mathbb{C})=\mathbf{g}_{x_{0}} \perp i \mathbf{g}_{x_{0}} .
$$

In addition the isomorphism of Lemma 5.2 maps $v \in \mathbf{g}_{x_{0}}$ to $-i v \in T_{x_{0}} \mathbb{X}^{3}$.

(b) In the spherical case $\mathbf{g} \cong s u(2) \times s u(2)$. Up to conjugation, $\mathbf{g}_{x_{0}} \cong$ $s u(2)$ is the subalgebra of diagonal matrices and $T_{x_{0}} \mathbb{X}^{3}$ is the set of antidiagonal elements (ie, matrices of the form $(a,-a)$ with $a \in s u(2)$ ). Hence isomorphism (5) is the decomposition of matrices of $s u(2) \times s u(2)$ as the sum of diagonal plus anti-diagonal elements. The isomorphism of Lemma 5.2 maps $(a, a) \in \mathbf{g}_{x_{0}}$ to $(a,-a) \in T_{x_{0}} \mathbb{X}^{3}$.

As an application we obtain:

Proposition 5.3 Let $\mathbb{X}^{2} \subset \mathbb{X}^{3}$ denote the geodesic hyperplane preserved by $\rho_{0}$ (tangent to $\left.\mathbb{R}^{2} \times 0\right)$. Then for every $(0, t) \in U, \rho_{(0, t)}$ preserves $\mathbb{X}^{2}$. 
Proof In the hyperbolic case we use the fact that $f_{g}$ is odd and $h_{g}$ is even. Hence $f_{g}(i t)$ is purely imaginary and $h_{g}(i t)$ is real. Thus $f_{g}(i t)$ is an infinitesimal translation tangent to $\mathbb{X}^{2}$ and $h_{g}(i t)$ is an infinitesimal rotation around a geodesic perpendicular to $\mathbb{X}^{2}$. This means that $f_{g}(i t)+h_{g}(i t)$ belongs to the Lie algebra of the isometry group of $\mathbb{X}^{2}$. In the spherical case, $\left(f_{g}(s), f_{g}(-s)\right)=\left(f_{g}(s),-f_{g}(s)\right)$ and $\left(h_{g}(s), h_{g}(-s)\right)=\left(h_{g}(s), h_{g}(s)\right)$, which also means that these elements belong to the Lie algebra tangent to the isometry group of $\mathbb{X}^{2}$.

\subsection{Infinitesimal properties of the section}

Let $\partial_{s} \rho: \pi_{1} M \rightarrow \mathbf{g}$ denote the cocycle defined by

$$
\left.g \mapsto \partial_{s}\left(\rho_{(s, t)}(g) \rho_{0}\left(g^{-1}\right)\right)\right|_{(s, t)=0} \quad \text { for every } g \in \pi_{1} M .
$$

We use the equivalent notation for $\partial_{t}$.

Lemma $\mathbf{5 . 4}$ (i) The cocycle $\partial_{s} \rho$ is valued on infinitesimal rotations.

(ii) The cocycle $\partial_{t} \rho$ is valued on infinitesimal translations.

(iii) Under the identification of Lemma 5.2, $\partial_{s} \rho=\partial_{t} \rho$. In addition, they are valued on the invariant plane $\mathbb{R}^{2} \times\{0\}$.

Proof Assertion (i) follows from Proposition 4.9. The remaining assertions follow easily from construction. For instance, in the hyperbolic case, $\partial_{s} \rho=$ $f_{g}^{\prime}(0)$ and $\partial_{t} \rho=-i f_{g}^{\prime}(0)$, because $h_{g}^{\prime}(0)=0$ ( $h_{g}$ is even). In the spherical case, $\partial_{s} \rho=\left(f_{g}^{\prime}(0), f_{g}^{\prime}(0)\right)$ and $\partial_{t} \rho=\left(f_{g}^{\prime}(0),-f_{g}^{\prime}(0)\right)$. (See the explanation after Lemma 5.2).

Definition 5.5 We define $\partial_{s} \partial_{s} \log \rho$ to be the chain in $C^{1}(M, \mathbf{g})$ such that $\forall g \in \pi_{1} M$,

$$
\left(\partial_{s} \partial_{s} \log \rho\right)(g)=\left.\frac{\partial^{2}}{\partial s^{2}} \log \left(\rho_{(s, t)}(g) \rho_{0}\left(g^{-1}\right)\right)\right|_{(s, t)=0} .
$$

We use the same definition for all other partial derivatives.

Proposition 5.6 There exists a choice of $p \in N i l$ and of the holonomy representation hol: $\pi_{1} \mathcal{O} \rightarrow$ Nil such that, if $q=\pi(p)$, then:

(i) $\partial_{s} \rho=z_{q}$,

(ii) The cochain $\partial_{s} \partial_{t} \log \rho$ is valued on infinitesimal translations along the invariant line $0 \times \mathbb{R}$ and equals to $c_{p}$. 
(iii) The translational part of $\partial_{s} \partial_{s} \log \rho$ and of $\partial_{t} \partial_{t} \log \rho$ vanish.

Proof The cocycle $z_{q} \in Z^{1}\left(M, \mathbb{R}^{2} \times 0\right)$ represents a non-zero element in cohomology. In addition, $\partial_{s} \rho$ is also non-zero in cohomology, because $w$ is locally a parametrization. Since $H^{1}\left(M, \mathbb{R}^{2} \times 0\right) \cong \mathbb{R}$, by composing the holonomy hol with an automorphism of $\mathrm{Nil}$ of the form

$$
\left(x_{1}, x_{2}, x_{3}\right) \mapsto\left(\lambda x_{1}, \lambda x_{2}, \lambda^{2} x_{3}\right), \quad \text { for all }\left(x_{1}, x_{2}, x_{3}\right) \in N i l,
$$

we have equality (i) up to coboundary. The choice of $q$ eliminates the indeterminacy of the coboundary.

To prove (ii), since $f_{g}^{\prime \prime}(0)=0$, in the hyperbolic case we have $\left(\partial_{s} \partial_{t} \log \rho\right)(g)=$ $-i h_{g}^{\prime \prime}(0)$ and in the spherical case $\left(\partial_{s} \partial_{t} \log \rho\right)(g)=\left(h_{g}^{\prime \prime}(0),-h_{g}^{\prime \prime}(0)\right)$. In both cases $\left(\partial_{s} \partial_{t} \log \rho\right)(g)$ is an infinitesimal translation with value $h_{g}^{\prime \prime}(0) \in 0 \times \mathbb{R}$.

From the second order terms in the expression $\varrho_{w}\left(g_{1} g_{2}\right)=\varrho_{w}\left(g_{1}\right) \varrho_{w}\left(g_{2}\right)$ we obtain:

$$
h_{g_{1}}^{\prime \prime}(0)+A d_{\rho_{0}\left(g_{1}\right)}\left(h_{g_{2}}^{\prime \prime}(0)\right)+\left[f_{g_{1}}^{\prime}(0), A d_{\rho_{0}\left(g_{1}\right)}\left(f_{g_{2}}^{\prime}(0)\right)\right]=h_{g_{1} g_{2}}^{\prime \prime}(0)
$$

(use for instance the Campbell-Hausdorff formula). Hence

$$
\partial_{s} \rho \cup \partial_{s} \rho=\delta\left(\partial_{s} \partial_{t} \log \rho\right) .
$$

Since $H^{1}(M, 0 \times \mathbb{R})=0$, we have that $c_{p}$ equals $\partial_{s} \partial_{t} \log \rho$ up to a coboundary. Again the indeterminacy of the coboundary is eliminated by choosing conveniently $p \in \pi^{-1}(q)$.

Finally to prove (iii), in the hyperbolic case $\left(\partial_{s}^{2} \log \rho\right)(g)=-\left(\partial_{t}^{2} \log \rho\right)(g)=$ $h_{g}^{\prime \prime}(0)$ and in the spherical case $\left(\partial_{s}^{2} \log \rho\right)(g)=\left(\partial_{t}^{2} \log \rho_{0}\right)(g)=\left(h_{g}^{\prime \prime}(0), h_{g}^{\prime \prime}(0)\right)$. In both cases, these are infinitesimal rotations.

\subsection{Compatibility with the holonomy}

In this subsection we prove property (iii) of Proposition 3.3; property (iv) being similar is not proved. We want to prove that for every $g \in \pi_{1} M$ :

$$
\lim _{\substack{s, t) \rightarrow 0 \\ s t \neq 0}} \Delta_{(s, t)}^{-1} \circ \rho_{(s, t)}(g) \circ \Delta_{(s, t)}=\operatorname{hol}(g)
$$

uniformly on compact subsets of $N i$ for the $\mathcal{C}^{1}$-topology.

Proof We fix $g \in \pi_{1} M$. We know that

$$
\exp _{x_{0}}^{-1}\left(\rho_{(s, t)}(g)\left(\Delta_{(s, t)}\left(x_{1}, x_{2}, x_{3}\right)\right)\right)
$$

is analytic on $(s, t)$ and on $\left(x_{1}, x_{2}, x_{3}\right)$. In addition: 
- the expression (6) is a multiple of $t$, because when $t=0, \rho_{(s, 0)}(g)$ fixes $x_{0}$ (Proposition 4.9), and

- the coefficient in $e_{3}$ of (6) is a multiple of $s t$, because when $s=0$, $\rho_{(0, t)}(g)$ preserves $\mathbb{X}^{2}=\exp _{x_{0}}\left(\mathbb{R}^{2} \times 0\right)=\exp _{x_{0}}\left\langle e_{1}, e_{2}\right\rangle$ (Proposition 5.3).

Thus it suffices to compute the first order terms of (6). More precisely, we write the expression (6) as follows:

$$
f_{1}(x,(s, t)) e_{1}+f_{2}(x,(s, t)) e_{2}+f_{3}(x,(s, t)) e_{3}
$$

for some analytic functions $f_{i}$ such that $f_{1}$ and $f_{2}$ are multiples of $t$ and $f_{3}$ is a multiple of $s t$. We want to prove that

$$
\left(\partial_{t} f_{1}(x, 0), \partial_{t} f_{2}(x, 0), \partial_{t} \partial_{s} f_{3}(x, 0)\right)=\operatorname{hol}(g)(x) .
$$

We notice that analyticity implies that the convergence is uniform on compact subsets for the $\mathcal{C}^{1}$-topology.

Corresponding to the basis $\left\{e_{1}, e_{2}, e_{3}\right\}$ for the sub-algebra $s u(2)=\mathbf{g}_{x}$ (ie, infinitesimal rotations), there is a basis for the space of infinitesimal translations $\left\{w_{1}, w_{2}, w_{3}\right\}$ via the isomorphism of Proposition 5.6. We have the following relations up to cyclic permutation of coefficients:

$$
\left[e_{1}, e_{2}\right]=e_{3},\left[w_{1}, w_{2}\right]=k e_{3},\left[e_{1}, w_{2}\right]=w_{3},\left[e_{1}, w_{1}\right]=0,
$$

where $k= \pm 1$ is the curvature of $\mathbb{X}^{3}$. In addition we have

$$
\Delta_{(s, t)}\left(x_{1}, x_{2}, x_{3}\right)=\exp \left(t x_{1} w_{1}+t x_{2} w_{2}+s t x_{3} w_{3}\right)\left(x_{0}\right) .
$$

If $\operatorname{hol}(g)$ is the multiplication by $\left(a_{1}, a_{2}, a_{3}\right) \in N i l$ composed with $\rho_{0}(\gamma)$, then by Proposition 5.6

$$
\rho_{s, t}(g)=\exp \left(a_{1}\left(s e_{1}+t w_{1}\right)+a_{2}\left(s e_{2}+t w_{2}\right)+a_{3} s t w_{3}+A\right) \rho_{0}(g)
$$

where $A$ are higher order terms (of order two multiplying $e_{1}, e_{2}, e_{3}, w_{1}, w_{2}$ and of order three multiplying $\left.w_{3}\right)$.

Since $\rho_{0}(g)\left(\Delta_{(s, t)}(x)\right)=\Delta_{(s, t)}\left(\rho_{0}(g)(x)\right)$, we may assume that $\rho_{0}(g)$ is trivial. We use the following notation

$$
\begin{aligned}
& R=s a_{1} e_{1}+s a_{2} e_{2} \\
& T=t\left(a_{1} w_{1}+a_{2} w_{2}+s a_{3} w_{3}\right) \\
& X=t\left(x_{1} w_{1}+x_{2} w_{2}+s x_{3} w_{3}\right)
\end{aligned}
$$

so that $\rho_{(s, t)}(g)=\exp (R+T+A)$ (we are assuming that $\rho_{0}(g)=\mathrm{Id}$ ) and $\Delta_{(s, t)}\left(x_{1}, x_{2}, x_{3}\right)=\exp (X)\left(x_{0}\right)$. Hence:

$$
\rho_{(s, t)}(g)\left(\Delta_{(s, t)}\left(x_{1}, x_{2}, x_{3}\right)\right)=\exp (R+T+A) \exp (X)\left(x_{0}\right) .
$$


By the Campbell-Hausdorff formula:

$$
\begin{aligned}
& \exp (R+T+A) \exp (X)=\exp \left(R+T+X+\frac{1}{2}[R+T, X]+A\right) \\
& \quad=\exp \left(T+X+\frac{1}{2}[R+T, X]-\frac{1}{2}[T+X, R]+A\right) \exp (R) \\
& =\exp (T+X+[R, X]+A) \exp (R),
\end{aligned}
$$

where $A$ is as above, because $[T, X]$ is an infinitesimal rotation of order two and $[R, T]$ is a translation but of order three. Since $\exp (R)\left(x_{0}\right)=x_{0}$, it follows that

$$
\rho_{(s, t)}(g)\left(\Delta_{(s, t)}\left(x_{1}, x_{2}, x_{3}\right)\right)=\exp (T+X+[R, X]+A)\left(x_{0}\right) .
$$

In addition $[R, X]=\left(a_{1} x_{2}-a_{2} x_{1}\right) s t w_{3}+O\left(s^{2} t\right)$, and property (iii) of Proposition 3.3 follows.

\section{Euclidean structures}

In this section we prove the part of Theorem 3.1 concerning Euclidean structures. We use the semi-direct product structure of the isometry group and its universal covering:

$$
\operatorname{Isom}^{+}\left(\mathbb{R}^{3}\right) \cong \mathbb{R}^{3} \rtimes S O(3), \quad \widetilde{\operatorname{Isom}^{+}}\left(\mathbb{R}^{3}\right) \cong \mathbb{R}^{3} \rtimes S U(2) .
$$

Definition 6.1 For $s$ in a neighborhood of the origin, we define the representation $\rho_{s}^{\prime}: \pi_{1} M \rightarrow \mathbb{R}^{3} \rtimes S U(2)$ as:

$$
\rho_{s}^{\prime}=\left(\partial_{t} \rho_{(s, 0)}, \rho_{(s, 0)}\right)
$$

Notice that $\rho_{s}^{\prime}$ is a representation because $\partial_{t} \rho_{(s, 0)}$ is a cocycle twisted by $\rho_{(s, 0)}$. In particular ROT $\circ \rho_{s}^{\prime}=\rho_{(s, 0)}$. The action of $\rho_{s}^{\prime}(g)$ on $\mathbb{R}^{3}$ is the following:

$$
v \mapsto \rho_{(s, 0)}(g)(v)+\partial_{t} \rho_{(s, 0)}(g) \quad \forall v \in \mathbb{R}^{3} \cong T_{x_{0}} \mathbb{X}^{3}
$$

Definition 6.2 We define the map $D_{s}^{\prime}: \widetilde{M} \rightarrow T_{x_{0}} \mathbb{X}^{3} \cong \mathbb{R}^{3}$ as

$$
D_{s}^{\prime}(x)=\left.\partial_{t} D_{(s, t)}(x)\right|_{t=0}
$$

Since $D_{(s, 0)}$ is the constant map $x_{0}$, the image of $D_{s}^{\prime}$ is contained in $T_{x_{0}} \mathbb{X}^{3}$.

The following proposition shows that $D_{s}^{\prime}$ is a developing map

Proposition 6.3 The map $D_{s}^{\prime}$ is $\rho_{s}^{\prime}$-equivariant and it is a local diffeomorphism for $s \neq 0$. 
The proof requires the following lemma.

Lemma 6.4 Let $\gamma:(-\varepsilon, \varepsilon) \rightarrow \mathbb{X}^{3}$ be a path such that $\gamma(0)=x_{0}$ and $\gamma^{\prime}(0)=$ $v \in T_{x_{0}} \mathbb{X}^{3}$. Then

$$
\rho_{s}^{\prime}(g)(v)=\left.\partial_{t}\left(\rho_{(s, t)}(g)(\gamma(t))\right)\right|_{t=0} .
$$

Proof By the chain rule:

$$
\begin{aligned}
\left.\partial_{t} \rho_{(s, t)}(g)(\gamma(t))\right|_{t=0}=\left.\partial_{t} \rho_{(s, t)}(g)\left(x_{0}\right)\right|_{t=0}+\rho_{0}(g)\left(\gamma^{\prime}(0)\right)= \\
\\
\partial_{t} \rho_{(s, 0)}(g)+\rho_{0}(g)(v)=\rho_{s}^{\prime}(g)(v) .
\end{aligned}
$$

Proof of Proposition 6.3 Equivariance of $D_{s}^{\prime}$ follows from deriving the following equality

$$
\rho_{(s, t)}(g)\left(D_{(s, t)}(x)\right)=D_{(s, t)}(g \cdot x)
$$

and applying Lemma 6.4.

Next we write $\Delta_{s}^{\prime}(x)=\left.\partial_{t} \Delta_{(s, t)}(x)\right|_{t=0}$. We have

$$
\Delta_{s}^{\prime}\left(x_{1}, x_{2}, x_{3}\right)=x_{1} e_{1}+x_{2} e_{2}+s x_{3} e_{3}
$$

Hence $\Delta_{s}^{\prime}$ is a diffeomorphsim for $s \neq 0$. We claim that

$$
\lim _{s \rightarrow 0}\left(\Delta_{s}^{\prime}\right)^{-1} \circ \rho_{s}^{\prime}(g) \circ \Delta_{s}^{\prime}=\operatorname{hol}(g) .
$$

The proof of this claim follows a scheme similar to the proof of Proposition 3.3 (iii) and deriving with respect to $t$ some of its equalities.

The construction of $D_{(s, t)}$ implies that $D_{s}^{\prime}$ can also be constructed by using bump functions. Hence $\left(\Delta_{s}^{\prime}\right)^{-1} \circ D_{s}^{\prime}$ converges to $\mathcal{D}_{0}$ uniformly on compact subsets, and the proposition follows.

\section{Deformation space and Dehn filling coefficients}

In this section we construct the deformation space Def, we define the Dehn filling coefficients and we study its behaviour. At the end of the section we prove Theorem B.

Definition 7.1 We define the deformation space Def as the open set

$$
\text { Def }=\left\{(s, \tau) \in \mathbb{R}^{2} \mid s \geq 0,(s, \tau) \text { in a neighborhood of } 0\right\}
$$

such that $(s, \tau)$ corresponds to the structure with parameters $(s, t)$ as follows:

- when $\tau>0$, it corresponds to the hyperbolic structure with $\tau=t^{2}$,

- when $\tau<0$, to the spherical structure with $\tau=-t^{2}$,

- when $\tau=0$, to the Euclidean structure with $t=0$. 


\subsection{Dehn filling coefficients}

We shall define the Dehn filling coefficients and prove that they induce an analytic map on $(s, \tau) \in$ Def:

$$
(p, q): \text { Def } \rightarrow \mathbb{R}^{2}
$$

We recall that in Subsection 4.2 we have chosen $l, m \in \pi_{1} M$ that generate a peripheral subgroup $\pi_{1} T^{2}$, so that $m$ is a meridian of $\Sigma$. We notice that since $l$ and $m$ commute, their holonomies have a common invariant geodesic.

Definition 7.2 For a geometric structure with holonomy $\rho_{(s, t)}$, we define $u \in$ $\mathbb{C}$ to be the complex length of $\rho_{(s, t)}(m)$ (ie, $\rho_{(s, t)}(m)$ is translation of length $\operatorname{Re}(u)$ composed with a rotation of angle $\operatorname{Im}(u)$ along the invariant geodesic). We also define $v \in \mathbb{C}$ as the complex length of $\rho_{(s, t)}(l)$.

The parameters $(u, v)$ are not uniquely defined. Besides $(u, v)$ we could choose any pair in the following set:

$$
\pm(u+2 \pi i \mathbb{Z}, v+2 \pi i \mathbb{Z}) .
$$

The choice of the sign depends on the orientation of the geodesic invariant by $\rho_{(s, t)}(l)$ and $\rho_{(s, t)}(m)$. We view $u(s, t)$ and $v(s, t)$ as analytic functions on $(s, t)$, hence they are unique if we fix the branch with $u(0,0)=\pi i$ and $v(0,0)=0$.

Definition 7.3 Given $(s, t) \in U,(p, q) \in \mathbb{R}^{2}$ are defined by the rule

$$
p u+q v=2 \pi i .
$$

This definition is equivalent to:

$$
\left.\begin{array}{l}
p \operatorname{Re} u+q \operatorname{Re} v=0 \\
p \operatorname{Im} u+q \operatorname{Im} v=2 \pi
\end{array}\right\}
$$

Proposition 7.4 If we fix the branch $p(0,0)=2$ and $q(0,0)=0$, then $(p, q)$ is an analytic map on $(s, \tau) \in$ Def.

Proof We start by describing $(u, v)$ as analytic maps on $(s, t)$ in the hyperbolic, spherical and Euclidean cases.

Let $w$ be the local parameter of Definition 4.3, and let $\varrho_{w}=\sigma(w)$, where $\sigma$ is the section in Lemma 4.6. By Lemmas 4.6 and 4.7 (v), there exists an odd analytic function $F$ with real coefficients such that

$$
\varrho_{w}(m)= \pm \exp \left((\pi+F(w)) e_{1}\right)= \pm \exp \left(F(w) e_{1}\right) \exp \left(\pi e_{1}\right) .
$$


Since $m$ and $l$ commute, by definition of $w$ we have:

$$
\varrho_{w}(l)= \pm \exp \left(w e_{1}\right) .
$$

In the hyperbolic case, $w=s-i t$ (see Subsection 4.3), hence:

$$
\left\{\begin{array}{l}
u_{H}=i(\pi+F(s-i t))=\operatorname{Im}(F(s+i t))+i(\pi+\operatorname{Re}(F(s+i t))) \\
v_{H}=i(s-i t)=t+i s
\end{array}\right.
$$

In the spherical case, we work in $X(M, S U(2)) \times X(M, S U(2))$ and we take $\left(w_{1}, w_{2}\right)=(s+t, s-t)$ (see also Subsection 4.3). Hence:

$$
\left\{\begin{array}{l}
u_{S}=(F(s+t)-F(s-t)) / 2+i(\pi+(F(s+t)+F(s-t)) / 2) \\
v_{S}=t+i s
\end{array}\right.
$$

In the Euclidean case the translational part is obtained by deriving with respect to $t$ when $t=0$ (see Section 6 ). Thus:

$$
\left\{\begin{array}{l}
u_{E}=F^{\prime}(s)+i(\pi+F(s)) \\
v_{E}=1+i s
\end{array}\right.
$$

Before showing that $(p, q)$ are well defined, we must notice that $\operatorname{Re}\left(u_{H}\right)$ and $\operatorname{Re}\left(u_{S}\right)$ are both multiples of $t=\operatorname{Re}\left(v_{H}\right)=\operatorname{Re}\left(v_{S}\right)$. Hence we redefine:

$$
\left\{\begin{array}{l}
\tilde{u}_{H}=\operatorname{Im}(F(s+i t)) / t+i(\pi+\operatorname{Re}(F(s+i t))) \\
\tilde{v}_{H}=1+i s \\
\tilde{u}_{S}=(F(s+t)-F(s-t)) /(2 t)+i(\pi+(F(s+t)+F(s-t)) / 2) \\
\tilde{v}_{S}=1+i s .
\end{array}\right.
$$

We keep $\tilde{u}_{E}=u_{E}$ and $\tilde{v}_{E}=v_{E}$. The system of equations (7) becomes

$$
\left.\begin{array}{l}
p \operatorname{Re} \tilde{u}+q \operatorname{Re} \tilde{v}=0 \\
p \operatorname{Im} \tilde{u}+q \operatorname{Im} \tilde{v}=2 \pi
\end{array}\right\}
$$

Since $\operatorname{Re} \tilde{v}=1, \operatorname{Im} \tilde{u}=\pi+O(s, t), \operatorname{Re} \tilde{u}=O(s, t)$ and $\operatorname{Im} \tilde{v}=s$, it is clear from this system of equations that $(p, q)$ is a well-defined analytic map on $(s, t)$ in every case (hyperbolic, Euclidean and spherical).

To show that $(p, q)$ is an analytic map on $(s, \tau) \in D e f$, we must check the following properties:

(i) $\tilde{u}_{H}(s, t)=\tilde{u}_{S}(s, i t)$.

(ii) $\tilde{u}_{H}(s, 0)=\tilde{u}_{S}(s, 0)=\tilde{u}_{E}(s)$

(iii) $\tilde{u}_{H}(s, t)$ and $\tilde{u}_{S}(s, t)$ are even on $t$.

These properties are obvious from construction. 


\subsection{The power expansion of $(p, q)$}

In this section we compute the power expansion of $(p, q)$. First we need the following proposition.

Proposition 7.5 $F(w)=a_{3} w^{3}+O\left(w^{5}\right)$, with $a_{3}>0$.

Lemma 7.6 $F^{\prime}(0)=0$.

Proof Using the notation of Lemma 4.4, $\alpha_{m}=\pi+F(w)$. In the same lemma it is proved that $d \alpha_{m}=0$, thus $F^{\prime}(0)=0$.

Proof of Proposition 7.5 We know that $F$ is an odd function with $F^{\prime}(0)=$ 0 . In the proof we use Theorem 3.1: there is a neigbhorhood $U \subset \mathbb{R}^{2}$ of the origin such that for every $(s, t) \in U$ with $s t \neq 0, \rho_{(s, t)}$ is the holonomy of a hyperbolic structure on $M$ with end of Dehn filling type. The structure at the end is described by $u$ and $v$.

We first show that $F$ is not constant by contradiction. If $F$ is constant, then $F \equiv 0$ because $F$ is odd, and $u \equiv \pi i$. This implies that all the structures on $U$ induce hyperbolic cone structures with cone angle $\pi$. This is impossible, because it implies that $\mathcal{O}$ is hyperbolic.

Let $2 n+1 \geq 3$ be the order of the first derivative such that $F^{(2 n+1)}(0) \neq 0$. We claim that $2 n+1=3$. Identifying $\mathbb{C} \cong \mathbb{R}^{2}$ via $w=s-t i$, the map $\left.F\right|_{U}$ is a branched covering of the open set $F(U) \subset \mathbb{C}$. It is branched at the origin with branching order $2 n+1$. We look at the inverse image of the real line $\left(\left.F\right|_{U}\right)^{-1}(\mathbb{R})$. It consists of $2 n+1$ curves passing through the origin. One of them is real, hence it corresponds to $t=0$ in $U$. The other $2 n$ curves, are contained in $\{(s, t) \in U \mid s t \neq 0\}$, hence they give geometric structures, except for the origin. Since the image of these curves is real, they correspond to cone structures.

The intersection of these $2 n$ curves with $\{(s, t) \in U \mid s t \neq 0\}$ has $4 n$ components, (each curve is divided into two when we remove the origin). Thus there are $n$ curves on the quadrant $\{(s, t) \in U \mid s>0, t>0\}$. If $n \geq 2$, then there would be at least two curves in the same quadrant. These two curves correspond to two families of structures with the same orientation and spin structure. In addition, when we parameter the curves from the origin, one of them has decreasing cone angle $\alpha_{m}=\pi+F(w)$, but the other one has increasing cone angle. This is not possible, because Schläfli's formula implies that the cone angles must decrease. Hence $n=1$ and $2 n+1=3$. 
Finally, the argument above gives $a_{3}>0$, because the branch of the first quadrant corresponds to decreasing volume.

We will determine the power expansion of $(p, q)$ by analyzing its behavior on the curves $s=0, \tau=-s^{2}$ and $\tau=0$.

Lemma 7.7 The Dehn filling coefficients $(p, q)$ induce a bijection between the segments $s=0$ and $p=2$;

Proof Since $F$ is odd, $F(i t)$ has zero real part. Hence, when $s=0, \tilde{u}_{H}(0, t)=$ $F(i t) /(i t)+i \pi$ and $\tilde{v}_{H}(0, t)=1$. Therefore in the hyperbolic case

$$
p=2, \quad q=-2 F(i t) /(i t)=2 a_{3} t^{2}+O\left(t^{4}\right)=2 a_{3} \tau+O\left(\tau^{2}\right) .
$$

In addition, $\tilde{u}_{S}(0, t)=F(t) / t+i \pi$ and $\tilde{v}_{S}(0, t)=1$. Thus in the spherical case

$$
p=2, \quad q=-2 F(t) / t=-2 a_{3} t^{2}+O\left(t^{4}\right)=2 a_{3} \tau+O\left(\tau^{2}\right),
$$

which is the same as equation (9) but for $\tau<0$.

Lemma 7.8 The Dehn filling coefficients $(p, q)$ induce a bijection between the curve $\tau=-s^{2}$ and the segment $p=2, q \leq 0$.

Proof Since the equation $\tau=-s^{2}$ is equivalent to $t=s$ in the spherical case, we have $\tilde{u}_{S}(s, s)=F(2 s) /(2 s)+i(\pi+F(2 s) / 2)$ and $v_{S}(s, s)=1+i s$. This gives the curve:

$$
p=2, \quad q=-F(2 s) / s=-8 a_{3} s^{2}+O\left(s^{4}\right)=8 a_{3} \tau+O\left(\tau^{2}\right)
$$

Hence the lemma is clear.

Remark The structures of Lemma 7.7 are transversely riemannian foliations. The structures of Lemma 7.8 are spherical and they are equipped with an isometric foliation of codimension 2 (in particular it is also transversely spherical). This comes from the fact that the equation $s=t$ implies that the parameter in Subsection 4.3 is $\left(w_{1}, w_{2}\right)=(2 s, 0)$. Hence the image of the holonomy representation is contained in $S U(2) \times \widetilde{O(2)}$, where $\widetilde{O(2)}$ is the lift of $O(2)<S O(3)$. Hence it is compatible with the isometric action of $\{1\} \times S^{1}<S U(2) \times S U(2)$.

Lemma 7.9 The Dehn filling coefficients map the half line $\tau=0$ bijectively to a half curve with power expansion:

$$
\left\{\begin{array}{l}
p=2+\frac{4 a_{3}}{\pi} s^{3}+O\left(s^{5}\right) \\
q=-6 a_{3} s^{2}+O\left(s^{4}\right)
\end{array}\right.
$$


Proof When $\tau=0, u_{E}=F^{\prime}(s)+i(\pi+F(s))$ and $v_{E}=1+i s$. Hence

$$
p=2 \pi /\left(\pi+F(s)-s F^{\prime}(s)\right) \text { and } q=-p F^{\prime}(s) .
$$

Since $F(s)=a_{3} s^{3}+O\left(s^{5}\right)$, the lemma is straightforward.

Definition 7.10 We define $g:(-\varepsilon, \varepsilon) \rightarrow \mathbb{R}$ to be a real function such that, for $q \geq 0, g(q)=2$, and for $q \leq 0, p=g(q)$ is the half curve of Lemma 7.9.

Corollary 7.11 We have the following power expansion:

$$
\left\{\begin{array}{l}
p=2+s\left(s^{2}+\tau\right)\left(\frac{4 a_{3}}{\pi}+O(s, \tau)\right) \\
q=2 a_{3}\left(\tau-3 s^{2}\right)+O\left(\tau s^{2}\right)+O\left(\tau^{2}\right)+O\left(s^{3}\right)
\end{array}\right.
$$

Proof By Lemmas 7.7 and 7.8, $p-2$ is a multiple of $s\left(\tau+s^{2}\right)$. The coefficient $\frac{4 a_{3}}{\pi}$ comes from Lemma 7.9. The power expansion of $q$ is straightforward from equation (9) and Lemma 7.9. We notice that $q$ has no coefficient in $\tau s$, by equation (10).

\subsection{The Whitney pleat}

In the next proposition we view $(p, q)$ as a function on $(s, \tau)$ defined not only on Def but in a neighborhood of the origin in $\mathbb{R}^{2}$.

Proposition 7.12 The map $(p(s, \tau), q(s, \tau))$ has a Whitney pleat at the origin, with folding curve $\tau=-9 s^{2}+O\left(s^{3}\right)$.

Proof Using the power expansion of Corollary 7.11, the Jacobian is:

$$
J(s, \tau)=\left|\begin{array}{cc}
p_{s} & p_{\tau} \\
q_{s} & q_{\tau}
\end{array}\right|=\frac{8 a_{3}^{2}}{\pi}\left(9 s^{2}+\tau\right)+O\left(\tau s^{2}\right)+O\left(\tau^{2}\right)+O\left(s^{3}\right)
$$

Hence $J=0$ is a curve with power expansion $\tau=-9 s^{2}+O\left(s^{3}\right)$. To show that there is a Whitney pleat with folding curve $J=0$, we compute the power expansion of $q$ restricted to this curve:

$$
\phi(s)=q\left(s,-9 s^{2}+O\left(s^{3}\right)\right)=-24 a_{3} s^{2}+O\left(s^{3}\right) .
$$

Since $\phi^{\prime \prime}(0)=-48 a_{3} \neq 0$, the proposition follows [22].

The image of the folding curve $J=0$ is a curve with power expansion:

$$
\left\{\begin{array}{l}
p=2-\frac{32 a_{3}}{\pi} s^{3}+O\left(s^{4}\right) \\
q=-\frac{24 a_{3}}{\pi} s^{2}+O\left(s^{3}\right)
\end{array}\right.
$$


Definition 7.13 We define $f:(-\varepsilon, \varepsilon) \rightarrow \mathbb{R}$ to be a real function such that, for $q \geq 0, f(q)=2$, and for $q \leq 0, p=f(q)$ is the image of the folding curve $J=0$, with $s \geq 0$.

Proof of Theorem B It is clear from Proposition 7.12 and Lemmas 7.7, 7.8 and 7.9. Notice that the restriction of $(p, q)$ to Def gives only half of the Whitney pleat, as in Figure 2. The curves that relevant in the proof of Theorem B are recalled in Figure 3.

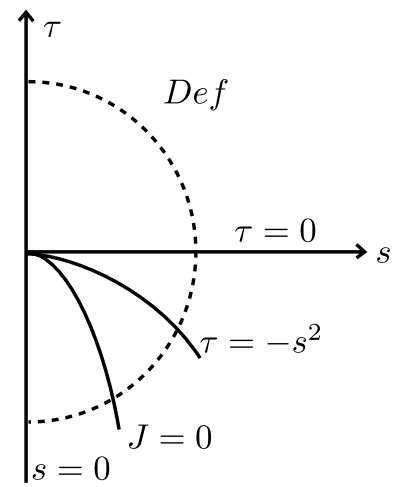

Figure 3: The curves in the proof of Theorem B. The folding curve is $J=0$, and it is mapped to $p=f(q)$. The curves $s=0$ and $\tau=-s^{2}$ are mapped to $p=2$. The segment $\tau=0$ is the Euclidean region, and it is mapped to $p=g(q)$

\section{The path of cone structures}

In this section we prove Propositions 1.3 and 1.4 by using the path of cone manifolds. We also prove the last statement of Theorem A concerning the limit when rescaling those cone manifolds.

Cone structures are determined by the equality $q=0$. From the power expansion of Corollary 7.11, it is clear that $q=0$ defines a curve in Def. This curve can be parametrized as:

$$
\tau=3 s^{2}+O\left(s^{3}\right) .
$$

Since $\tau>0$ those structures are hyperbolic. The other coefficient is $p=$ $2+\frac{16}{\pi} a_{3} s^{3}+O\left(s^{4}\right)$. Thus the cone angle is:

$$
\alpha=2 \pi / p=\pi-8 a_{3} s^{3}+O\left(s^{4}\right)
$$


and therefore the path of cone structures is:

$$
\left\{\begin{array}{l}
s(\alpha)=\frac{1}{2} \sqrt[3]{\frac{\pi-\alpha}{a_{3}}}+O\left(|\pi-\alpha|^{2 / 3}\right) \\
t(\alpha)=\frac{\sqrt{3}}{2} \sqrt[3]{\frac{\pi-\alpha}{a_{3}}}+O\left(|\pi-\alpha|^{2 / 3}\right) .
\end{array}\right.
$$

Next we compute some magnitudes of those cone manifolds using the parameter $s$. The length of the singular set is

$$
\operatorname{length}\left(\Sigma_{\alpha}\right)=\operatorname{Re}(v)=t=\sqrt{\tau}=\sqrt{3} s+O\left(s^{2}\right) .
$$

Thus, by Schläfli's formula the variation of volume is

$$
d \operatorname{vol}\left(C_{\alpha}\right)=-\frac{1}{2} \operatorname{length}\left(\Sigma_{\alpha}\right) d \alpha=\left(12 \sqrt{3} a_{3} s^{3}+O(4)\right) d s .
$$

Therefore

$$
\operatorname{vol}\left(C_{\alpha}\right)=3 \sqrt{3} a_{3} s^{4}+O(5)
$$

Proof of Proposition 1.3 Straightforward from the computations above.

Below we use that

$$
l_{0}=\lim _{\pi \rightarrow \alpha} \frac{\operatorname{length}\left(\Sigma_{\alpha}\right)}{(\pi-\alpha)^{1 / 3}}=\frac{\sqrt{3}}{2 a_{3}^{1 / 3}} .
$$

Proof of Proposition 1.4 We use the descriptions of the curves $p=f(q)$ and $p=g(q)$ when $q<0$ given in previous section. First at all, the parametrization of $p=f(q)$ when $q<0$ has a power expansion described in equation (11) (Subsection 7.3). Therefore:

$$
\lim _{q \rightarrow 0^{-}} \frac{2-f(q)}{|q|^{3 / 2}}=\frac{\sqrt{2}}{3 \sqrt{3} \pi \sqrt{a_{3}}}=\frac{4}{9 \sqrt[4]{3} \pi} l_{0}^{3 / 2}
$$

The curve $p=g(q)$ has a power expansion described in Lemma 7.9, when $q<0$. Thus:

$$
\lim _{q \rightarrow 0^{-}} \frac{g(q)-2}{|q|^{3 / 2}}=\frac{\sqrt{2}}{3 \sqrt{3} \pi \sqrt{a_{3}}}=\frac{4}{9 \sqrt[4]{3} \pi} l_{0}^{3 / 2},
$$

which proves Proposition 1.4.

The following proposition finishes the proof of Theorem A.

Proposition 8.1 When $\alpha \rightarrow \pi^{-}$, the cone manifolds $C_{\alpha}$ re-scaled by ( $\pi-$ $\alpha)^{-1 / 3}$ converge to the orbifold basis of the Seifert fibration of $\mathcal{O}$. In addition, when they are re-scaled by $(\pi-\alpha)^{-1 / 3}$ in the horizontal direction and by $(\pi-\alpha)^{-2 / 3}$ in the vertical one, they converge to $\mathcal{O}$. 
Proof Let $\pi: N i l \rightarrow \mathbb{R}^{2}$ denote the projection of the Riemannian fibration of Nil, ie, $\pi\left(x_{1}, x_{2}, x_{3}\right)=\left(x_{1}, x_{2}\right)$. The developing map of the transverse structure of the Seifert fibration of $\mathcal{O}$ is

$$
\pi \circ \mathcal{D}_{0}: \widetilde{\mathcal{O}} \rightarrow \mathbb{R}^{2}
$$

where $\mathcal{D}_{0}: \widetilde{\mathcal{O}} \rightarrow N i l$ is the developing map of the $N i l$-structure.

Let $(s(\alpha), t(\alpha))$ denote the path of cone structures. Since $t(\alpha)$ has order $(\pi-$ $\alpha)^{-1 / 3}$, to prove the first part of the proposition is sufficient to show that

$$
\lim _{\alpha \rightarrow \pi^{-}} \frac{1}{t(\alpha)} \exp _{x_{0}}^{-1} \circ D_{(s(\alpha), t(\alpha))}=\pi \circ D_{0}
$$

uniformly on compact subsets of $\widetilde{M}$. To prove this limit, we write

$$
\frac{1}{t(\alpha)} \exp _{x_{0}}^{-1} \circ D_{(s(\alpha), t(\alpha))}=\frac{1}{t(\alpha)} \exp _{x_{0}}^{-1} \circ \Delta_{(s(\alpha), t(\alpha))} \circ \Delta_{(s(\alpha), t(\alpha))}^{-1} \circ D_{(s(\alpha), t(\alpha))} .
$$

By the proof of Theorem 3.1, $\Delta_{(s(\alpha), t(\alpha))}^{-1} \circ D_{(s(\alpha), t(\alpha))} \rightarrow \mathcal{D}_{0}$. In addition

$$
\frac{1}{t(\alpha)} \exp _{x_{0}}^{-1} \circ \Delta_{(s(\alpha), t(\alpha))}\left(x_{1}, x_{2}, x_{3}\right)=\left(x_{1}, x_{2}, s(\alpha) x_{3}\right) .
$$

Since $s(\alpha) \rightarrow 0$, the limit is clear. Notice that since $s(\alpha)$ has also order $(\pi-\alpha)^{-1 / 3}$, the second part of the proposition follows easily.

\section{An example}

We consider the orbifold $\mathcal{O}$ described as follows. Its underlying space is the lens space $L(4,1)$, which we view as the result of Dehn surgery on the trivial knot in $S^{3}$ with surgery coefficient 4 . We view this trivial knot as one component of the Whitehead link, and the branching locus $\Sigma$ is precisely the other component of the link (see Figure 4).

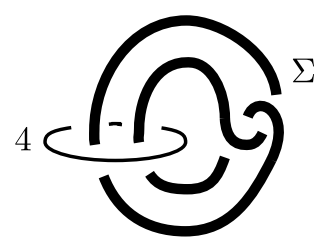

Figure 4: The surgery description of the orbifold $\mathcal{O}$.

It is well known that the Whitehead link has a Montesinos fibration. This induces an orbifold Seifert fibration of $\mathcal{O}$. By looking at the basis of this fibration and its Euler number, one can check that $\mathcal{O}$ has $N i l$ geometry. 
We want to compute the limit $l_{0}$ of Propositions 1.3 and 1.4. To do it, we consider the variety of characters of $M=\mathcal{O}-\Sigma$. The manifold $M$ is a punctured torus bundle over the circle, with homological monodromy $\left(\begin{array}{ll}1 & 4 \\ 1 & 5\end{array}\right)$. Thus its fundamental group admits a presentation

$$
\pi_{1}(\mathcal{O}-\Sigma)=\left\langle a, b, m \mid m a m^{-1}=a b, m b m^{-1}=b(a b)^{4}\right\rangle
$$

where $m$ is the meridian of the branching locus. We also choose $l=a b a^{-1} b^{-1}$, so that $l, m$ generate a peripheral group. The variety of characters can be easily computed by using the methods of [16]. To compute $l_{0}$, we do not need the whole variety of characters, but only its projection to the plane generated by the variables $x=I_{m}$ and $y=I_{l}$. This projection can be computed by means of resultants and it gives the planar curve:

$$
(y-2)^{3}+x^{2}\left(64-16 x^{2}+x^{4}+(y-2)\left(32-5 x^{2}\right)+(y-2)^{2}\left(7-5 y^{2}\right)\right)=0
$$

The projection of $\chi_{0}$ to this curve has coordinates $(x, y)=(0,2)$.

Using the results of Section 7, we write

$$
y=2 \cosh (i w / 2) \quad \text { and } \quad x=2 \cosh (i(\pi+F(w)) / 2) .
$$

Since $F(w)=a_{3} w^{3}+O\left(w^{5}\right)$, we have that

$$
y=2-w^{2} / 2+O\left(w^{4}\right) \quad \text { and } \quad x=-a_{3} w^{3}+O\left(w^{5}\right) .
$$

By replacing those values in the the equation of the curve above we obtain:

$$
-(w / 2)^{6}+\left(a_{3} w^{3}\right)^{2} 64+O\left(w^{8}\right)=0 .
$$

Hence $a_{3}=2^{-6}$. Since $l_{0}=\sqrt{3} /\left(2 a_{3}^{1 / 3}\right)$, this implies that

$$
\lim _{\alpha \rightarrow \pi^{-}} \frac{\operatorname{length}\left(\Sigma_{\alpha}\right)}{(\pi-\alpha)^{1 / 3}}=l_{0}=2 \sqrt{3} \quad \text { and } \quad \lim _{q \rightarrow 0^{-}} \frac{2-f(q)}{|q|^{3 / 2}}=\frac{8 \sqrt{2}}{3 \sqrt{3} \pi} .
$$

\section{Cohomology computations}

The aim of this section is to prove:

$$
H^{1}\left(M, \mathbb{R}^{2} \times 0\right) \cong \mathbb{R} \quad \text { and } \quad H^{1}(M, 0 \times \mathbb{R})=0 .
$$

First we need to compute the homology of the orbifold $\mathcal{O}$, that can be defined as follows. Let $K$ be a triangulation of the underlying space of $\mathcal{O}$ compatible with $\Sigma$. It induces a triangulation $\tilde{K}$ of $\tilde{\mathcal{O}} \cong N i l$. Let $V$ be a $\pi_{1} \mathcal{O}$-module. We consider the following chain and cochain complexes:

$$
\begin{aligned}
& C_{*}(K ; V)=V \otimes_{\pi_{1} \mathcal{O}} C_{*}(\tilde{K} ; \mathbb{Z}) \\
& C^{*}(K ; V)=\operatorname{Hom}_{\pi_{1} \mathcal{O}}\left(C_{*}(\tilde{K} ; \mathbb{Z}), V\right)
\end{aligned}
$$


The homology of $C_{*}(K ; V)$ is denoted by $H_{*}(\mathcal{O} ; V)$ and the cohomology of $C^{*}(K ; V)$ by $H^{*}(\mathcal{O} ; V)$. From the differential point of view, $H^{*}(\mathcal{O} ; V)$ is the cohomology of the $V$-valued differential forms on $\widetilde{\mathcal{O}} \cong N i l$ which are $\pi_{1} \mathcal{O}_{-}$ equivariant. The same construction holds for $\Sigma$ and for a tubular neighborhood $\mathcal{N}(\Sigma)$.

We shall apply the Mayer Vietoris exact sequence to the pair $(M, \mathcal{N}(\Sigma))$, so that $M \cup \mathcal{N}(\Sigma)=\mathcal{O}$. We first compute the cohomology of $\mathcal{O}$.

Lemma 10.1 Let $V$ be either $\mathbb{R}^{2} \times 0$ or $0 \times \mathbb{R}$. There is a natural isomorphism $H^{*}(\mathcal{O}, V) \cong H^{*}\left(\pi_{1} \mathcal{O}, V\right)$.

Proof Let $P \rightarrow \mathcal{O}$ be a finite regular covering such that $P$ is a manifold. Let $\Gamma$ be the group of deck transformations of the covering. There is a natural isomorphism

$$
H^{*}(\mathcal{O}, V) \cong H^{*}(P, V)^{\Gamma}
$$

(See [2] for instance). We also have a natural isomorphism

$$
H^{*}\left(\pi_{1} \mathcal{O}, V\right) \cong H^{*}\left(\pi_{1} P, V\right)^{\Gamma} .
$$

Since $P$ is an aspherical manifold, there is another natural isomorphism

$$
H^{*}\left(\pi_{1} P, V\right)^{\Gamma} \cong H^{*}(P, V)^{\Gamma} .
$$

Hence the lemma follows by composing the three isomorphisms. Notice that since $C_{*}(\tilde{K} ; \mathbb{Z})$ is an acyclic $\pi_{1} \mathcal{O}$-module, there is a natural map $H^{*}\left(\pi_{1} \mathcal{O}, V\right) \rightarrow$ $H^{*}(\mathcal{O}, V)$, by homology theory, and that it is the composition of the three isomorphisms.

Lemma $10.2 H^{0}\left(\mathcal{O}, \mathbb{R}^{2} \times 0\right) \cong 0$ and $H^{1}\left(\mathcal{O}, \mathbb{R}^{2} \times 0\right) \cong \mathbb{R}$.

Proof Since $H^{0}\left(\mathcal{O}, \mathbb{R}^{2} \times 0\right) \cong H^{0}\left(\pi_{1} \mathcal{O}, \mathbb{R}^{2} \times 0\right) \cong\left(\mathbb{R}^{2} \times 0\right)^{\pi_{1} \mathcal{O}}$, this group is zero because the unique element of $\mathbb{R}^{2} \times 0$ invariant by $\pi_{1} \mathcal{O}$ is zero.

To compute $H^{1}\left(\mathcal{O}, \mathbb{R}^{2} \times 0\right)$ we use the regular covering $P \rightarrow \mathcal{O}$ of the previous proof, with deck transformation group $\Gamma$, and the isomorphism $H^{1}\left(P, \mathbb{R}^{2} \times\right.$ $0)^{\Gamma} \cong H^{1}\left(\mathcal{O}, \mathbb{R}^{2} \times 0\right)$. Since the image of $\phi_{0}$ is finite, we may assume that $\pi_{1} P<\operatorname{ker} \phi_{0}$. Hence the action of $\pi_{1} P$ on $\mathbb{R}^{2} \times 0$ is trivial and

$$
H^{*}\left(P, \mathbb{R}^{2} \times 0\right) \cong \operatorname{Hom}\left(H_{*}(P, \mathbb{R}), \mathbb{R}^{2} \times 0\right) .
$$

The manifold $P$ can be assumed to be a $S^{1}$-bundle over $T^{2}$ with non-trivial Euler number $e \neq 0$. In particular,

$$
\pi_{1} P \cong\left\langle t, \alpha, \beta \mid[t, \alpha]=[t, \beta]=1,[\alpha, \beta]=t^{e}\right\rangle
$$


Thus the projection $P \rightarrow T^{2}$ induces a isomorphism $H_{1}(P, \mathbb{R}) \cong H_{1}\left(T^{2}, \mathbb{R}\right)$ and:

$$
H^{1}\left(P, \mathbb{R}^{2} \times 0\right) \cong \operatorname{Hom}\left(H_{1}\left(T^{2}, \mathbb{R}\right), \mathbb{R}^{2} \times 0\right) \cong M_{2 \times 2}(\mathbb{R}) .
$$

where $M_{2 \times 2}(\mathbb{R})$ denotes the ring of $2 \times 2$ matrices with real coefficients. In this isomorphism the action of $\Gamma$ translates in $M_{2 \times 2}(\mathbb{R})$ as the linear action by conjugation of $\phi_{0}(\Gamma) \subset O(2)$. Since $\phi_{0}(\Gamma)$ is dihedral, $H^{1}\left(P, \mathbb{R}^{2} \times 0\right)^{\Gamma} \cong \mathbb{R}$.

With a similar argument one can prove:

Lemma $10.3 H^{*}(\mathcal{O} ; 0 \times \mathbb{R}) \cong 0$.

Corollary $10.4 H^{*}(M ; 0 \times \mathbb{R}) \cong 0$.

Proof We apply the Mayer-Vietoris exact sequence to the pair $(\mathcal{N}(\Sigma), M)$, where $\mathcal{N}(\Sigma)$ is a tubular neighborhood of $\Sigma$, so that $\mathcal{N}(\Sigma) \cup M=\mathcal{O}$ and $\mathcal{N}(\Sigma) \cap M \simeq T^{2}$. By Lemma 10.3, we have an isomorphism:

$$
H^{*}(M ; 0 \times \mathbb{R}) \oplus H^{*}(\mathcal{N}(\Sigma) ; 0 \times \mathbb{R}) \cong H^{*}\left(T^{2} ; 0 \times \mathbb{R}\right) .
$$

Since the meridian $m$ belongs to $\pi_{1} M$ and $\rho_{0}(m)$ acts non-trivially on $0 \times \mathbb{R}$, it follows that $H^{0}\left(T^{2} ; 0 \times \mathbb{R}\right) \cong H^{0}\left(\pi_{1} T^{2} ; 0 \times \mathbb{R}\right) \cong(0 \times \mathbb{R})^{\pi_{1} T^{2}} \cong 0$. By duality $H^{2}\left(T^{2} ; 0 \times \mathbb{R}\right) \cong 0$, and by Euler characteristic, $H^{1}\left(T^{2} ; 0 \times \mathbb{R}\right) \cong 0$.

Lemma 10.5 $H^{1}(M ; s u(2)) \cong \mathbb{R}$. In particular $H^{1}\left(M ; \mathbb{R}^{2} \times 0\right) \cong \mathbb{R}$.

Proof We apply a Mayer-Vietoris argument to the pair $(M, \mathcal{N}(\Sigma))$. Since $M \cup \mathcal{N}(\Sigma)=\mathcal{O}$ and $M \cap \mathcal{N}(\Sigma) \simeq T^{2}$, we have an exact sequence:

$$
H_{1}\left(T^{2}, s u(2)\right) \stackrel{i_{1} \oplus i_{2}}{\longrightarrow} H_{1}(M, s u(2)) \oplus H_{1}(\mathcal{N}(\Sigma), s u(2)) \stackrel{j_{1}-j_{2}}{\longrightarrow} H_{1}(\mathcal{O}, s u(2))
$$

where $i_{1}, i_{2}, j_{1}$ and $j_{2}$ are the natural maps induced by inclusion. Notice that $j_{1} \circ i_{1}=j_{2} \circ i_{2}$ by exactness. We have divided the proof in several steps.

(1) $H_{1}\left(T^{2}, s u(2)\right) \cong \mathbb{R}^{2}$ and $\left\{d \alpha_{l}, d \alpha_{m}\right\}$ is a basis for $H_{1}\left(T^{2}, s u(2)\right)$.

This follows from the local properties of the variety of representations $R\left(T^{2}, S U(2)\right)$. See [16], for instance.

(2) $j_{2} i_{2}\left(d \alpha_{l}\right)=j_{1} i_{1}\left(d \alpha_{l}\right) \neq 0$. In particular it is a basis for $H_{1}(\mathcal{O}, s u(2))$. The proof that $j_{1} i_{1}\left(d \alpha_{l}\right) \neq 0$ uses the same argument as the proof of Lemma 4.4. More precisely, since $\pi \circ \operatorname{hol}(l)$ is a nontrivial translation, the Kronecker pairing between the cocycle $z_{q}=$ TRANS $_{q} \circ \pi \circ$ hol and $d \alpha_{l}$ does not vanish (Prop. 9.6 from [17]). Thus $d \alpha_{l} \neq 0$ when viewed in $H^{1}\left(\pi_{1} \mathcal{O}, s u(2)\right)$. Since $H^{1}(\mathcal{O}, s u(2)) \cong H^{1}\left(\pi_{1} \mathcal{O}, s u(2)\right) \cong \mathbb{R}$, by Lemmas 10.2 and 10.3 , it is clear that this element is a basis. 
(3) $i_{2}\left(d \alpha_{m}\right)=0$.

This follows easily from the computation of $H_{1}(\mathcal{N}(\Sigma), S U(2))$, because $m$ has order two, and therefore it is rigid (see [16] for details).

(4) $i_{1}: H_{1}\left(T^{2}, s u(2)\right) \rightarrow H_{1}(M, s u(2))$ has rank one.

Since this map is Poincaré dual to $H_{1}(M, \partial M, s u(2)) \rightarrow H_{1}\left(T^{2}, s u(2)\right)$, this follows from the long exact sequence of the pair $(M, \partial M)$ and Step 1.

(5) $i_{1}\left(d \alpha_{m}\right)=0$.

The proof is by contradiction. Assume that $i_{1}\left(d \alpha_{m}\right) \neq 0$. Then by Step 4 , $i_{1}\left(d \alpha_{l}\right)=\lambda i_{1}\left(d \alpha_{m}\right)$ for some $\lambda \in \mathbb{R}$. In addition, since $i_{2}\left(d \alpha_{m}\right)=0$ :

$$
j_{1} i_{1}\left(d \alpha_{l}\right)=\lambda j_{1} i_{1}\left(d \alpha_{m}\right)=\lambda j_{2} i_{2}\left(d \alpha_{m}\right)=0
$$

which contradicts Step 2.

(6) $H_{1}(M, s u(2)) \cong \mathbb{R}$.

By the previous steps $i_{1} \oplus i_{2}$ has rank one. The map $j_{1}-j_{2}$ has also rank one, because $H_{1}(\mathcal{O}, s u(2)) \cong \mathbb{R}$ and $j_{1}-j_{2}$ is surjective by Step 2. A standard computation shows that $\operatorname{dim}_{\mathbb{R}}\left(H_{1}(\mathcal{N}(\Sigma), S U(2))\right)=1$. Therefore $H_{1}(M, s u(2)) \cong \mathbb{R}$.

This finishes the proof of the lemma.

Acknowledgement This research was partially supported by MCYT through grant BFM2000-0007.

\section{References}

[1] L Ben Abdelghani, Espace des représentations du groupe d'un noeud dans un groupe de Lie, Thesis U. de Bourgogne (1998)

[2] G E Bredon, Introduction to compact transformation groups, Pure and Applied Mathematics, Vol. 46. Academic Press, New York-London (1972)

[3] M Boileau, J Porti, Geometrization of 3-orbifolds of cyclic type, Astérisque 272 (2001)

[4] M Boileau, B Leeb, J Porti, Uniformization of small 3-orbifolds, C. R. Acad. Sci. Paris Sér. I Math. 332 (2001) 57-62

[5] M Boileau, B Leeb, J Porti, Geometrization of 3-dimensional orbifolds, Part I: Geometry of cone manifolds, preprint (2002)

[6] M Boileau, B Leeb, J Porti, Geometrization of 3-dimensional orbifolds, preprint (2002) 
[7] R D Canary, D B A Epstein, P Green, Notes on notes of Thurston, from: "Analytical and Geometric Aspects of Hyperbolic Space" (D B A Epstein, editor) London Math. Soc. Lecture Notes Ser. 111 Cambridge Univ. Press, Cambridge (1987) 3-92

[8] D Cooper, C Hodgson, S Kerchkoff, Three dimensional Orbifolds and Cone Manifolds, Mathematical Society of Japan Memoirs 5 (2000)

[9] M Culler, Lifting representations to covering groups, Adv. in Math. 59 (1986) 64-70

[10] M Culler, P Shalen, Varieties or group representations and splittings of 3manifolds, Ann. of Math. 117 (1984) 401-476

[11] M Heusener, J Porti, E Suárez, Regenerating singular hyperbolic structures from Sol, J. Differential Geom. 59 (2001) 439-478

[12] C Hodgson, Degeneration and Regeneration of Hyperbolic Structures on ThreeManifolds, Thesis, Princeton University (1986)

[13] C Hodgson, S Kerckhoff, Rigidity of hyperbolic cone-manifolds and hyperbolic Dehn Surgery, J. Diff. Geom. 48 (1998) 1-59

[14] S Kojima, Deformations of hyperbolic 3-cone-manifolds, J. Diff. Geom. 49 (1998) 469-516

[15] A Lubotzky, A R Magid, Varieties of representations of finitely generated groups, Mem. of the Amer. Math. Soc. 58 (1985)

[16] J Porti, Torsion de Reidemeister pour les Variétés Hyperboliques, Mem. Amer. Math. Soc. 128 (1997)

[17] J Porti, Regenerating hyperbolic and spherical cone structures from Euclidean ones, Topology 37 (1998) 365-392

[18] E Suárez, Poliedros de Dirichlet de 3-variedades cónicas y sus deformaciones, Thesis, U. Complutense de Madrid (1998)

[19] P Scott, The geometries of 3-manifolds, Bull. London Math. Soc. 15 (1983) 401-487

[20] W P Thurston, The Geometry and Topology of 3-manifolds, Princeton Math. Dept. (1979)

[21] W P Thurston, Three-dimensional geometry and topology, Vol. 1. (S Levy, editor) Princeton University Press, Princeton, NJ (1997)

[22] H Whitney, On singularities of mappings of Euclidean spaces. I. Mappings of the plane into the plane, Ann. of Math. 62 (1955) 374-410 